\title{
Timoshenko beam models for the coupled analysis of building response to tunnelling
}

\author{
Andrea Franza ${ }^{\mathrm{a}, *}$, Sinan Acikgoz ${ }^{\mathrm{b}}$, Matthew J. DeJong ${ }^{\mathrm{c}}$ \\ ${ }^{a}$ ETSI Caminos, Canales y Puertos, Universidad Politécnica de Madrid, Calle Profesor Aranguren, 28040, Madrid, Spain. \\ ${ }^{b}$ Department of Engineering Science, University of Oxford, Parks Road, OX1 3PJ, Oxford, United Kingdom. \\ ${ }^{c}$ Department of Civil and Environmental Engineering, University of California, Berkeley, CA 94720, United States.
}

\begin{abstract}
Timoshenko beam theory and the limiting tensile strain method are implemented into the computer program ASRE for the coupled analysis of building response to tunnelling using an elastic two-stage analysis method. A framework to characterise an equivalent Timoshenko beam is proposed that is consistent with previous works. More specifically, buildings are represented through equivalent anisotropic beam models in terms of axial, shear and bending cross-sectional stiffnesses, as well as the offset between the axis and the ground surface. Firstly, model predictions are compared with centrifuge results of tunnelling-induced distortions of building models with façade openings. Then, simple beams with varying Young's modulus to shear modulus ratios are analysed. Results detail tunnelling-induced displacements, internal forces, and deformations, as well as bending and diagonal strains of the Timoshenko beams; the way in which both axis-ground offset and shear flexibility level alter the tunnel-structure interaction mechanism was quantified. For damage assessments, an improved approach is proposed to estimate bending and diagonal strains. Finally, to enable practical implementation, design charts that relate both the relative soil-structure stiffness parameters (that account for both bending and shear contributions) and the foundation plan size ratio to the deflection ratio modification factors are given.
\end{abstract}

Keywords: Tunnelling, Ground movements, Soil-Structure Interaction, Timoshenko beam, Building Response, Damage estimation

1 Highlights

- Modelling tunnel-structure-interaction with Timoshenko beams founded on elastic continuum.

- Effects of shear flexibility quantified in terms of displacements and deformations.

- The offset between beam axis the ground level can impact the interaction problem.

- The 'direct strain based approach' was proposed to infer maximum building strains.

- Design charts with bending and shear structural stiffness as well as foundation shape ratio.

\footnotetext{
${ }^{*}$ Corresponding author

Email address: andreafranza@gmail.com (Andrea Franza)
} 


\section{Introduction}

There is an increasing demand in urban areas for underground constructions requiring the excavation of new tunnels. Tunnelling results in ground movements and, as the results of tunnel-structure interaction (TSI), these movements can deform and displace surface structures and, potentially, lead to damage. Consequently, during design stages of new tunnels, the effects of tunnelling on surface structures with varying structural characteristics need to be assessed.

Preliminary assessment of the effects of TSI is possible through greenfield ground movements and the modification factor approach (Franzius et al., 2006; Mair et al., 1996). Alternatively, two-stage methods may also be used while accounting for superstructure and foundation characteristics (Deck and Singh, 2012; El Kahi et al., 2018; Elkayam and Klar, 2019; Franza and DeJong, 2019; Son, 2015). For listed structures and scenarios associated with a high potential for damage, refined numerical modelling has the capability to predict cracking patterns (Bloodworth and Houlsby, 2017; Boldini et al., 2018; Fu et al., 2018; Yiu et al., 2017). Alternatively, equivalent structural models may be adopted for TSI analyses to decrease computational costs (Pickhaver et al., 2010; Losacco et al., 2014). In particular, engineers have often investigated the effects of the structural stiffness on TSI focusing on the value of equivalent bending stiffness (Giardina et al., 2015; Franzius et al., 2006; Haji et al., 2018a), or the equivalent shear stiffness (Son and Cording, 2005). Consequently, design charts for soil-structure interaction effects typically refer to either bending stiffness (Franzius et al., 2006; Mair, 2013), or shear stiffness values (Son and Cording, 2005).

This paper implements an equivalent Timoshenko beam on an elastic continuum model for the analysis of tunnel-structure interaction (TSI). The objective of this paper is to illustrate the influence of (i) relative shear flexibility and (ii) offset between the beam axis and ground level on the structural response. Within this context, structural deformations, internal forces, and tensile strains within the beam models are investigated. In particular, structural tensile strains are inferred from either the internal forces of the Timoshenko beam or the deformation parameters measured along the structure axis. Finally, the findings are used to formulate modified design charts for an improved estimation of deflection ratio modification factors.

\section{Background}

\subsection{Greenfield movements}

When structures and foundations are not present nearby to the tunnel excavation, tunnelling-induced vertical $\left(u_{z}\right)$ and horizontal $\left(u_{x}\right)$ greenfield movements at the surface can be estimated using the empirical methods. Equation (1) is adopted in clays (Peck, 1969; O'Reilly and New, 1982), while the modified Gaussian curve can be used for the settlement trough obtained in sands (Franza et al., 2019a; Marshall et al., 2012).

$$
u_{z}=-u_{z, \max } \exp \left(-\frac{x^{2}}{2 i^{2}}\right) \quad u_{x}=u_{z} \frac{x}{z_{t}}
$$

where $x$ and $z$ are respectively the horizontal and vertical spatial coordinates, $u_{z, \max }$ is the maximum settlement value, $z_{t}$ is the tunnel axis depth, $i=K z_{t}$ is the horizontal distance of the settlement trough inflection point to the tunnel centreline, and $K$ is the surface width parameter. In clays, $K=0.5$ and $u_{z, \max }=1.25 R^{2} V_{l, t} /(100 i)$ (Mair et al., 1993), where $R$ is the tunnel radius and $V_{l, t}$ is the tunnel volume loss parameter. 


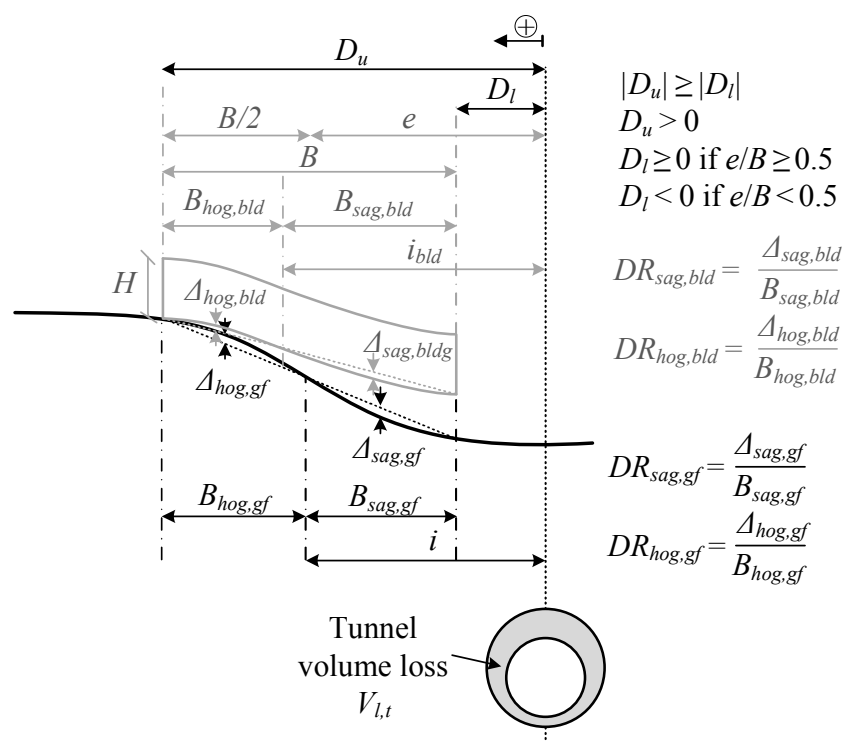

Figure 1: Nomenclature for the flexural TSI problem

\subsection{Tunnelling-induced structure deformations and the role of soil-structure interaction}

Semi-flexible structures react to tunnelling- and excavation-induced ground displacements. To quantify the effect of structural stiffness on the deformations induced by tunnelling, Potts and Addenbrooke (1997) proposed the deflection ratio modification factors, $M^{D R, s a g}$ an $M^{D R, h o g}$, as follows

$$
M^{D R, \text { sag } / \text { hog }}=\frac{D R_{\text {sag } / \text { hog }, \text { bld }}}{D R_{\text {sag } / \text { hog,gf }}}
$$

where building deflection ratios are $D R_{\text {sag,bld }}$ and $D R_{\text {hog,bld }}$ (defined with respect to the lengths $B_{\text {sag,bld }}$ and $B_{\text {hog,bld }}$ based on the building settlement profile; depending on the structure inflection point, $i_{b l d}$ ), and deflection ratios of the greenfield settlement trough are $D R_{s a g, g f}$ and $D R_{h o g, g f}$ (depending on the building length $B_{\text {sag,gf }}$ and $B_{h o g, g f}$ in the sagging and hogging zones of the greenfield settlement trough; determined by the greenfield inflection point position, $i$ ) are defined in Figure 1.

Similar to the DRs, modification factors for the average horizontal strain (Dimmock and Mair, 2008) can be defined as follows

$$
M^{\text {sh,sag/hog }}=\frac{\varepsilon_{h, \text { sag } / \text { hog }, \text { bld }}}{\varepsilon_{h, \text { sag } / \text { hog }, g f}}
$$

where $\varepsilon_{h, s a g / h o g, b l d}$ and $\varepsilon_{h, s a g / h o g, g f}$ are computed, respectively, within $B_{\text {sag/hog,bld }}$ and $B_{\text {sag/hog,gf }}$.

TSI can vary the shape of the structure settlement curve with respect to greenfield ground movements (i.e. the structure inflection point, $i_{b l d}$, can be different than the inflection point of the greenfield settlement trough, $i$ ), as shown in Figure 1 (Farrell et al., 2014; Potts and Addenbrooke, 1997). To quantify this, the modification factors $M^{B, s a g}$ and $M^{B, h o g}$ for the sagging and hogging region length are defined as follows

$$
M^{B, \text { sag } / \text { hog }}=\frac{B_{\text {sag } / \text { hog, bld }}}{B_{\text {sag } / \text { hog,gf }}}
$$

To estimate the impact of the structure stiffness on $M^{D R}$ for both tunnelling and deep-excavations, Mair 
(2013) proposed the following relative bending stiffness factors

$$
\rho_{\text {sag } / \text { hog }}=\frac{E I}{E_{s} B_{\text {sag/hog,gf }}^{3} L}=\frac{E I^{*}}{E_{S} B_{\text {sag } / \text { hog, } g f}^{3}}
$$

where $E I$ is the bending stiffness of the superstructure (in $\mathrm{kN} \mathrm{m}^{2}$ ), $E I^{*}$ is $E I$ per running metre (in $\mathrm{kN} \mathrm{m}^{2} / \mathrm{m}$ ), $E_{s}$ is a soil Young's modulus representative of the soil affected by the excavation, $L$ is the longitudinal length of the building in the tunnel axis direction. As discussed later in the text, Equation (5) accounts for the structure stiffness exclusively in terms of bending stiffness $E I$.

Depending on the shape of the building settlement profile, modified relative stiff factors $\rho_{\text {sag }}^{\prime}$ and $\rho_{\text {hog }}^{\prime}$ in Equation (6) were defined by Franza et al. (2019b) for Euler-Bernoulli beams with pure bending deformations relatively to the structure lengths $B_{\text {sag,bld }}$ and $B_{\text {hog,bld }}$ in the sagging and hogging zones (rather than for the greenfield values $B_{\text {sag,gf }}$ and $B_{h o g, g f}$ ).

$$
\rho_{\text {sag } / \text { hog }}^{\prime}=\frac{E I^{*}}{E_{s} B_{\text {sag } / \text { hog,bld }}^{3}}=\frac{\rho_{\text {sag } / \text { hog }}}{\left(M^{B, \text { sag } / \text { hog }}\right)^{3}}
$$

\subsection{Traditional damage assessments: displacement (DBA) and modification factor (MBA) based approaches}

For both greenfield and SSI risk assessments using the limiting strain method (Boscardin and Cording, 1989; Mair et al., 1996), the maximum tensile strain in a given structure cross-section, $\varepsilon_{\max }$, is the greater value between the total bending strain at the beam edge, $\varepsilon_{b t}$, and the total shearing strain at the fibre with the maximum shear stress, $\varepsilon_{d t}$. Using Mohr's circle of strain,

$$
\varepsilon_{b t}=\varepsilon_{b}+\varepsilon_{h} \quad \varepsilon_{d t}=\left(\varepsilon_{b}^{\prime}+\varepsilon_{h}\right)\left(\frac{1-v}{2}\right)+\sqrt{\left(\varepsilon_{b}^{\prime}+\varepsilon_{h}\right)^{2}\left(\frac{1+v}{2}\right)^{2}+\varepsilon_{d}^{2}}
$$

where $\varepsilon_{b}$ is the bending strain at the extreme fibre, $\varepsilon_{b}^{\prime}$ and $\varepsilon_{d}$ are the bending and diagonal strain at the fibre with the maximum shear stress, respectively, $\varepsilon_{h}$ is the horizontal axial strain at the beam axis, and $v$ is the beam Poisson's ratio. Greenfield maximum tensile strains, $\varepsilon_{\max , g f}$, of the target building are estimated by considering a fully-flexible structure, while TSI maximum tensile strains, $\varepsilon_{\text {max,tsi }}$, are estimated using the actual structural stiffness.

In current practice, strains $\left(\varepsilon_{b}, \varepsilon_{d}\right.$ and $\left.\varepsilon_{h}\right)$ are estimated from displacements of the foundations in the 'displacement based approach' (DBA). To achieve this, the deflection ratios and the average horizontal strains are associated to strains using the deflection ratio DR in Equation (8) (Burland et al., 1977) and assuming $\varepsilon_{h}$ as the average horizontal axial strain within the sagging/hogging region (Mair et al., 1996)

$$
\varepsilon_{b}=\frac{D R}{\left(\frac{B_{r}}{12 t}+\frac{3}{2 t B_{r}} \frac{\alpha E I_{0}}{\beta G A_{0}}\right)} \quad \varepsilon_{b}^{\prime}=0 \quad \varepsilon_{d}=\frac{D R}{\left(1+\frac{B_{r}^{2}}{18} \frac{\beta G A_{0}}{\alpha E I_{0}}\right)} \quad \varepsilon_{h}=\varepsilon_{a v g}
$$

where the length of the building portion in the sagging or hogging zone is $B_{r}=\left[B_{\text {sag,bld }} ; B_{\text {hog,bld }}\right], D R=$ $D R_{b l d}$, and $\varepsilon_{h}=\varepsilon_{h, b l d}$.

Alternatively, the 'modification factor based approach' (MBA) computes the maximum strains from the modification factor and the greenfield displacement profiles using Equation (8) (i.e. $B_{r}=\left[B_{\text {sag,gf }} ; B_{h o g, g f}\right]$, $\left.D R=M^{D R} D R_{g f}, \varepsilon_{h}=M^{\varepsilon_{h}} \varepsilon_{h, g f}\right)$.

Interestingly, Equation (8) was estimated assuming that the structure portion in the sagging and hogging regions with a given $D R$ behaves as a simply supported beam Timoshenko with zero curvature or bending 
moment at the edges. However, for a Timoshenko beam, both shear and bending contribute to the second derivative of the settlement profile (which is not directly proportional to the curvature); consequently, the inflection point used to identify $D R$ is not necessarily a point of zero curvature or bending moment.

\section{Proposed elastic models}

In this paper, the Timoshenko beam model and the limiting tensile strain method are implemented into the computer program ASRE (Franza and DeJong, 2019) for the coupled structure response to excavations using a two-stage analysis method. Greenfield ground movements, input for the coupled analyses may be estimated using either empirical methods, simulations, or experimental/field data.

\subsection{Geometry and assumptions}

The considered 'target structure' is a building transverse to the new tunnel and resting on a half-space continuum subjected to tunnelling-induced ground movements. This structure is modelled as an equivalent Timoshenko beam with cross-anisotropic behaviour (independent values for Young's modulus $E$, shear modulus $G$, and Poisson's ratio $v$ ). The geometry, nomenclature, and adopted conventions are shown in Figure 2. The cross-sectional properties and Young's and shear moduli of the beam elements are chosen to represent the structural characteristics of the target building. In this study, the displacement compatibility is assumed between the soil and the structure (i.e. separation and relative soil-structure sliding are not allowed), which is reasonable at low volume losses.

The influence of the offset between the structure 'axis' and ground level, $d_{a}$, is also investigated (see Figure 2). The 'axis' corresponds to the cross-sectional weighted centroid for both homogeneous and composite structures. The 'axis' is the location at which the applied axial loads do not result in the beam deflection. This also coincides with the location of the neutral axis for pure bending. For instance, a solid wall with a rectangular cross-section has its axis at mid-height, whereas a masonry wall with openings founded on a concrete strip footing would have an axis below the mid-height (see Figure 3(b)). Thus, the beam axis is a characteristic of the structure and can differ from the neutral axis, which depends on the applied loads. In this paper, a single value of $d_{a}$ was adopted as a weighted average of $d_{a}$ along the structure, as shown in Figure 2.

Elastic models are solved considering two different cases: the beam axis located at the ground level (labelled 'GL' solutions; $d_{a}=0$ ) and the beam axis located at its estimated height above the surface (labelled 'DA' solutions; $d_{a}>0$ ). Note that for GL solutions, greenfield tunnelling-induced forces are applied at the beam axis, whereas for DA solutions these forces are applied at the bottom fibres of the equivalent beam. To account for foundation elements parallel to the tunnel (i.e. perpendicular to the building plane under consideration), decoupled springs may be adopted, as later discussed.

\subsection{Equilibrium equation}

The finite element method (FEM) is used to solve the TSI problem numerically. For the GL solutions (Franza and DeJong, 2019), the equilibrium equation of the system, written in matrix form, is

$$
\mathbf{K u}=\mathbf{q} ; \quad \mathbf{K}=\mathbf{S}+\mathbf{K}_{\mathbf{s}} ; \quad \mathbf{q}=\mathbf{p}+\mathbf{K}_{\mathbf{s}} \mathbf{u}^{c a t}
$$

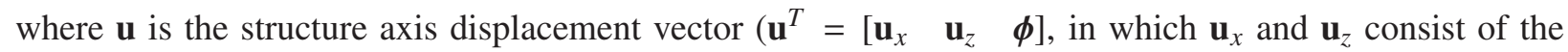
translational displacements along $x$ and $z$, respectively, whereas $\phi$ contains rotations of the finite element

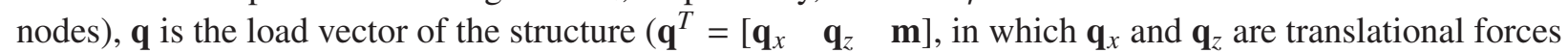
whereas $\mathbf{m}$ is the vector of applied bending moments), $\mathbf{u}^{\text {cat }}$ is the greenfield surface displacement due to 


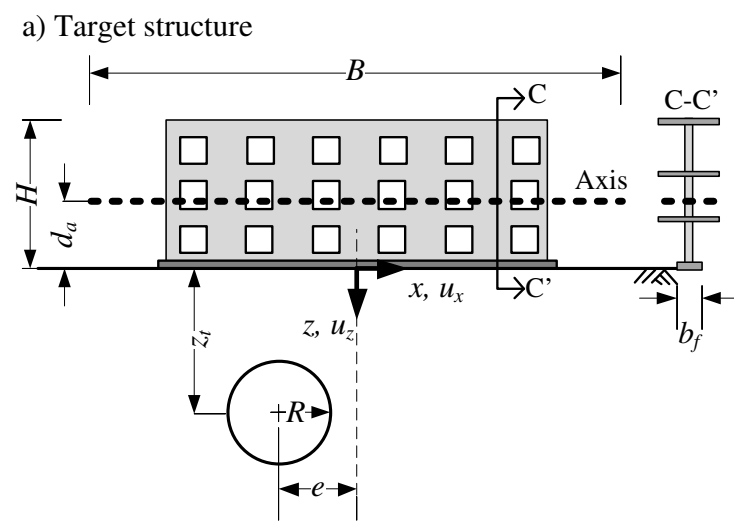
b) Equivalent Timoshenko beam (TIM) or Euler-Bernoulli beam (EB) on the elastic continuum
DA Solution
GL Solution
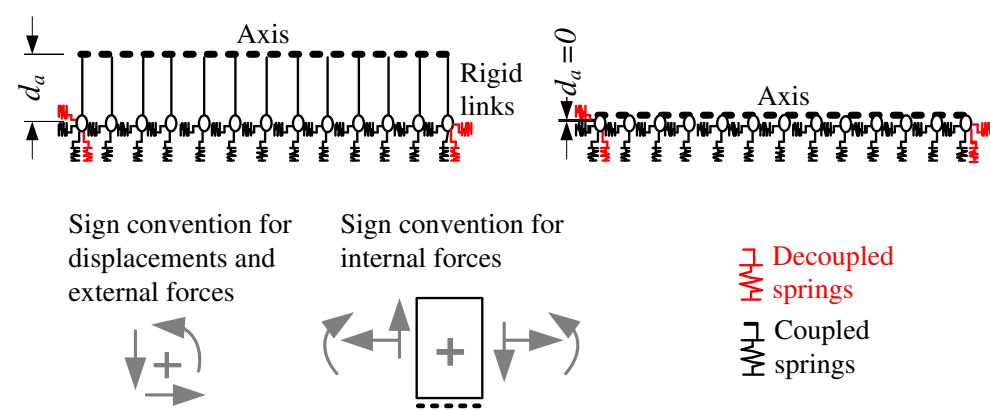

$$
\begin{aligned}
& \text { 구 Decoupled } \\
& \text { springs } \\
& \text { 굴 Coupled } \\
& \text { 소 springs }
\end{aligned}
$$

Figure 2: Tunnel-structure interaction: (a) geometry of the problem; (b) DA and GL solutions implementing equivalent beams.
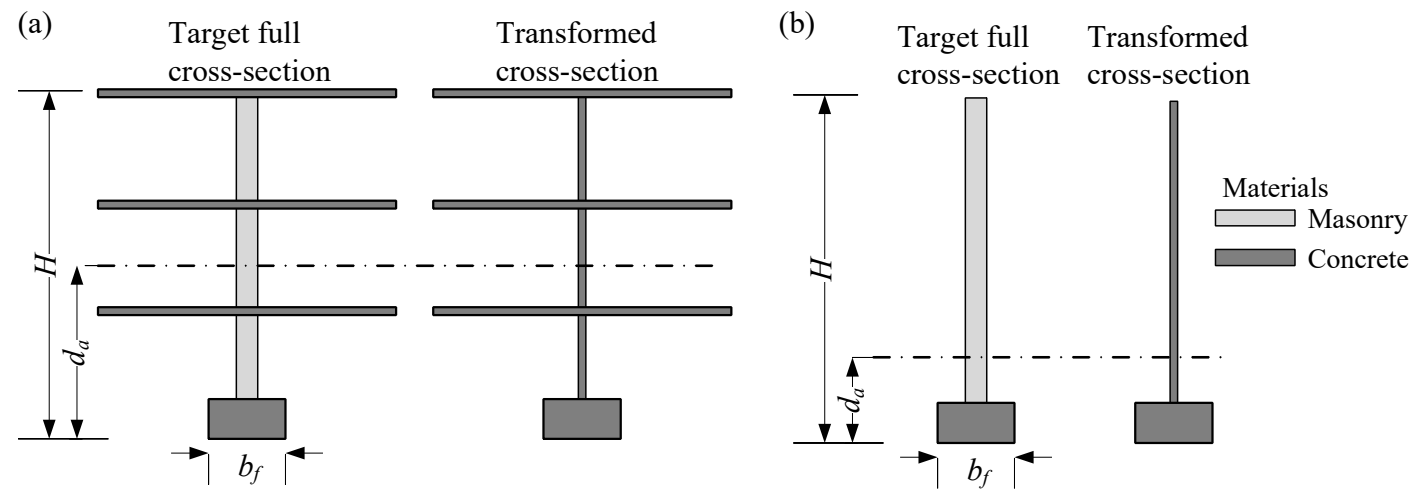

Figure 3: Transformed cross-section method: (a) frame with infill walls and (b) bearing wall with strip footing.

tunnelling (a generic greenfield displacement field can be the input to the model), $\mathbf{p}$ is the external loading vector of the structure (assumed equal to zero in this paper), $\mathbf{S}$ is the structure stiffness matrix, $\mathbf{K}_{\mathbf{s}}$ is the stiffness matrix of the soil equal to the inverse of the soil flexibility matrix defined with the elastic integrated forms of Mindlin's solutions given by Vaziri et al. (1982). Typically, springs of the soil continuum model are 'coupled'; the soil flexibility matrix consists of both diagonal and off-diagonal terms. Therefore, a force (the resultant of a stress distribution on a rectangular area) applied in correspondence of a spring results in both vertical and horizontal displacements of all springs located on the surface of the half-space. The term 'decoupled' is used when off-diagonal terms are neglected while describing the behaviour as individual spring elements.

For the DA solutions, the offset between the axis of the simple beam and ground level $\left(d_{a}\right)$ is implemented considering [i] linear relationships between beam axis and ground both in terms of displacements and forces, [ii] that the structure stiffness matrix $\mathbf{S}$ is given for forces and displacements with respect to its axis, and [iii] that the soil stiffness matrix $\mathbf{K}_{\mathbf{s}}$ and tunnelling-induced forces $\mathbf{K}_{\mathbf{s}} \mathbf{u}^{c a t}$ are given with respect to the ground level (i.e. structure bottom fibre). If primed symbols are used for the ground level reference 
system, then for the $i^{\text {th }}$ node

$$
\begin{aligned}
& u_{z}^{\prime}=u_{z} ; u_{x}^{\prime}=u_{x}+\phi d_{a} \\
& q_{z}^{\prime}=q_{z} ; q_{x}^{\prime}=q_{x} ; m=q_{x}^{\prime} d_{a}
\end{aligned}
$$

The equilibrium equation for DA solutions can be written as follows

$$
\mathbf{K}^{*} \mathbf{u}=\mathbf{q}^{*} ; \quad \mathbf{K}^{*}=\mathbf{S}+\mathbf{K}_{\mathbf{s}}{ }^{*} ; \quad \mathbf{q}^{*}=\mathbf{p}+\left(\mathbf{K}_{\mathbf{s}} \mathbf{u}^{c a t}\right)^{*}
$$

where $\mathbf{K}_{\mathbf{s}}{ }^{*}$ and $\left(\mathbf{K}_{\mathbf{s}} \mathbf{u}^{\text {cat }}\right)^{*}$ are the soil stiffness matrix and the vector of tunnelling-induced forces, respectively, at the structure axis reference system; $\mathbf{K}_{\mathbf{s}}{ }^{*}$ and $\left(\mathbf{K}_{\mathbf{s}} \mathbf{u}^{\text {cat }}\right)^{*}$ were obtained from $\mathbf{K}_{\mathbf{s}}$ and $\left(\mathbf{K}_{\mathbf{s}} \mathbf{u}^{\text {cat }}\right)$ using Equations (10a) and (10b) (Cheung and Nag, 1968).

\subsection{Equivalent beam models: axis location and stiffness values}

In this paper, the term 'total stiffness' refers to the contribution of axial, bending and shear stiffness $\left(E A, E I\right.$, and $\left.G A_{s}\right)$, which are independently controlled. An equivalent cross-anisotropic Timoshenko beam is adopted to represent the building by imposing Equation (12)

$$
E_{m} A_{m}=E A \quad E_{m} I_{m}=E I \quad G_{m} A_{s m}=G A_{s} \quad v_{m}=v \quad b_{m}=b_{f} \quad d_{a, m}=d_{a}
$$

where $E$ and $G$ are the Young's and shear moduli, $v$ is the Poisson's ratio, $I$ is the second moment of area, $A$ and $A_{s}=k A$ are the cross-sectional area and the shear area, respectively, related by the shear factor $\kappa, b_{f}$ is the cross-sectional width of the foundation strip footing. For a rectangular cross-section, $k=(10+10 v) /(12+11 v)$. The model properties are referred to with the subscript $m$.

For the target structure, $d_{a}$ is firstly estimated by neglecting the presence of openings. Then, the crosssectional stiffness values are computed as

$$
E A=\lambda E A_{0} \quad E I=\alpha E I_{0} \quad G A_{s}=\beta G A_{s 0}
$$

where $E A_{0}, E I_{0}$, and $G A_{s, 0}$ corresponds to the full-cross section whereas the presence of openings is accounted in terms of reduction factors $(\lambda, \alpha, \beta)$. For composite structures (composed of two or more materials), $E A_{0}, E I_{0}, G A_{s, 0}$ may be estimated by adopting the transformed-section method, as shown in Figure 3 (Kassimali et al., 1986). Reduction factors are computed with the strip method proposed by Pickhaver et al. (2010) with respect to the structure axis, while assuming that the axial factor is obtained as for the shear stiffness factor with no influence of the height-to-length ratio.

The link between the geometry and material characteristics of the equivalent beams and the properties of the target structure is detailed along with practical considerations in Appendix A.

\subsection{Damage assessment: direct Strain Based Approach (DSBA)}

The direct strain based approach (DSBA) is proposed in this paper, for both greenfield and SSI analyses, to overcome possible errors due to the simplifying assumptions of Equation (8) and, thus, DBA and MBA. At each cross-section, tunnelling-induced strains of the target structure are directly calculated (from either measured deformations of the target building or internal forces of the equivalent beam) using Equation (14). A summary of the implemented approaches and corresponding equations is given by Table 1 . 


$$
\varepsilon_{b}=\chi_{m} t=\frac{M_{m} t}{E I_{0} \alpha} \quad \varepsilon_{b}^{\prime}=\chi_{m} s=\frac{M_{m} s}{E I_{0} \alpha} \quad \varepsilon_{d}=\frac{c \gamma_{m}}{2}=\frac{c V_{m}}{2 \kappa A_{0} G \beta} \quad \varepsilon_{h}=\varepsilon_{a x i s, m}=\frac{N_{m}}{E A_{0} \lambda}
$$

where $\chi_{m}$ is the beam curvature, $\gamma_{m}$ is the beam engineering shear strain, $\varepsilon_{a x i s, m}$ is the beam axis strain, and the axial force, $N_{m}$, shear force, $V_{m}$, and bending moment, $M_{m}$ are the internal forces measured at the equivalent Timoshenko beam axis (see Figure 2(b) for sign convention of the internal forces). Also, $k$ and $c$ are the shear correction and the average shear stress corrective factors, which depend on the cross-sectional geometry, $t=\left[d_{a} ;-\left(H-d_{a}\right)\right]$ is the distance between the neutral axis and the extreme fibre experiencing tensile strain due to bending, and $s$ is the vertical distance (defined as positive downward) between the axis and the fibre where $\varepsilon_{d t}$ is calculated. For a rectangular cross-section, $c=3 / 2$ at the beam axis and $s=0$; different cross-sections may be considered by adopting different $c$ and $s$ values.

Note that Equation (14) can be used to infer strains from the deformation parameters $\chi, \gamma, \varepsilon_{\text {axis }}$ measured during experimental testing and field monitoring works. As shown by Ritter (2017); Ritter et al. (2019), these deformation parameters can be approximately inferred using the method proposed by Cook (1994) from the corner displacements of each bay.

In this paper, for the DSBA approach, displacements, deformation parameters, and internal forces that correspond to $\varepsilon_{\max , t s i}$ and $\varepsilon_{\max , g f}$ are calculated, respectively, by using the actual equivalent beam Young's modulus of $E_{m}$ and a low value of $E_{m}$, while the ratio $E_{m} / G_{m}$ is kept constant.

Table 1: Methods for calculating the maximum tensile strain from either greenfield or SSI analyses

\begin{tabular}{llll}
\hline & \multicolumn{1}{c}{ Input parameter } & Source of input parameters & Formula \\
\hline DSBA & Deformations / forces & Displacements along the entire building axis & Eq. (7), (14) \\
\cline { 2 - 4 } DBA & DRs and axial strains in sagging/hogging regions & $\begin{array}{l}\text { Displacements at the building axis } \\
\text { within the sagging and hogging portions }\end{array}$ & Eq. (7), (8) \\
\cline { 2 - 4 } MBA & DRs and axial strains in sagging/hogging regions & Greenfield displacements and modification factors & Eq. (7), (8) \\
\cline { 2 - 5 }
\end{tabular}

\section{Comparison with centrifuge data}

The proposed solution is compared with experimental results obtained from centrifuge testing at $75 \mathrm{~g}$ (Ritter et al., 2018, 2017) to validate the numerical models; the rectangular buildings were oriented with their long direction perpendicular to the tunnel axis, and consisted of four perimeter walls with strip footings and two intermediate interior walls (parallel to the tunnel axis) with no footings. In the following, model dimensions and results are reported in prototype scale. The tunnel (diameter $D=6.2 \mathrm{~m}$, cover-to-diameter ratio $C / D=1.3$, depth to tunnel axis $z_{t}=11.3 \mathrm{~m}$ ) was excavated in dense dry sand with soil relative density $I_{d}=90 \%$. The continuum solutions require the definition of greenfield surface movements, geotechnical model parameters and equivalent simple beam characteristics. Volume losses of $V_{l, t} \leq 2 \%$ were considered; accordingly, a representative Young's modulus $E_{s}=45 \mathrm{MPa}$ and a Poisson's ratio $v_{s}=0.25$ were assumed for the continuum. Tunnelling-induced vertical and horizontal displacements were set equal to empirical curves curve-fitted to greenfield centrifuge test results. Half of the structure (split along the longitudinal direction) was modelled with an equivalent Timoshenko beam, assuming $E=0.8 \mathrm{GPa}$ and isotropic behaviour $E / G=2.6$. The cross-sectional axial, bending, and shear stiffness were evaluated accounting for the presence of openings (opening ratio, $O$, varying between 20 and $40 \%$ of the façade area) with the strip method (Pickhaver et al., 2010); the characteristics of the target structures are summarised in Table 2.

By assuming plane-strain greenfield soil displacement field and neglecting the interaction between front and rear parts of the soil-structure system, the structure was modelled as a single façade (perpendicular to the 
Table 2: Façade characteristics of the centrifuge test series (prototype scale).

\begin{tabular}{|c|c|c|c|c|c|c|c|c|c|}
\hline $\begin{array}{c}\text { Label } \\
\text { Test }\end{array}$ & $\begin{array}{c}B \\
(\mathrm{~m})\end{array}$ & $\begin{array}{c}e / B \\
(-)\end{array}$ & $\begin{array}{c}O \\
(\%)\end{array}$ & $\begin{array}{c}E A^{\dagger} \\
(\mathrm{N})\end{array}$ & $\begin{array}{c}E I \dagger \\
\left(\mathrm{Nm}^{2}\right)\end{array}$ & $\begin{array}{c}G A_{s} \dagger \\
(\mathrm{N})\end{array}$ & $\begin{array}{r}v \\
(-)\end{array}$ & $\begin{array}{c}d_{a} \\
(\mathrm{~m})\end{array}$ & $\begin{array}{l}b_{f} \\
(\mathrm{~m})\end{array}$ \\
\hline SR-A & 15.0 & 0 & 20 & $1.5710^{9}$ & $5.2710^{9}$ & $3.0910^{8}$ & 0.30 & 2.5 & 0.8 \\
\hline SR-B & 15.0 & 0.8 & 20 & $1.5710^{9}$ & $5.2710^{9}$ & $3.0910^{8}$ & 0.30 & 2.5 & 0.8 \\
\hline SR-C & 15.0 & 0.5 & 20 & $1.5710^{9}$ & $5.2710^{9}$ & $3.0910^{8}$ & 0.30 & 2.5 & 0.8 \\
\hline SR-D & 15.0 & 0.8 & 40 & $1.2410^{9}$ & $4.3810^{9}$ & $2.4310^{8}$ & 0.30 & 2.5 & 0.8 \\
\hline SR-E & 19.5 & 0.5 & 20 & $1.5710^{9}$ & $6.8410^{9}$ & $4.0110^{8}$ & 0.30 & 2.5 & 0.8 \\
\hline SR-F & 19.5 & 0.5 & 40 & $1.2210^{9}$ & $5.6110^{9}$ & $3.1010^{8}$ & 0.30 & 2.5 & 0.8 \\
\hline
\end{tabular}

tunnel). For the sake of simplicity, the effects of the foundation scheme were investigated by implementing two soil stiffness matrix for the continuum: ' $\mathrm{C} 1$ ' considering only the soil beneath the long strip footings (perpendicular to the tunnel) with coupled springs; ' $\mathrm{C} 2$ ' modelling the soil beneath the long strip footings with coupled springs as well as the soil beneath half of the two end walls (parallel to the tunnel) using additional decoupled springs (see the external nodes of the Timoshenko beam in Figure 2(b)). The stiffness values of these decoupled springs were estimated for a soil-foundation contact surface of $0.8 \mathrm{~m} \times 3 \mathrm{~m}$.

Predictions of the model beam are compared with the centrifuge measurements of the façade at the target structure axis (a vertical distance from the ground level $d_{a}=2.5 \mathrm{~m}$ ) in terms of tunnelling-induced vertical and horizontal displacements, modification factors $M^{D R}$, as well as structure deformation parameters. Shear strains $\gamma$ and curvature $\chi$ are compared for both GL (dashed lines) and DA (solid lines) solutions in Figures 4 and 5; the effects of $\mathrm{C} 1$ (black lines) and $\mathrm{C} 2$ (grey lines) on the deformations of the beam model are also illustrated in these figures. For comparison, in Figure 5, the curvature profiles associated with the GL solution of a fully flexible EB beam are displayed. Finally, displacements and modification factors are provided in the supplemental data for the soil model C2 (Figures S1-S2).

Regarding settlements and modification factors, the equivalent Timoshenko beams (calibrated using the strip method) could reasonably predict the level of the deflection ratio modification factors. For instance, the DA solutions captured the relatively stiff response of the central structure SR-A (due to the coupled between bending of the structure and soil horizontal springs), as well as the horizontal shift of the eccentric buildings due to the tilt of the whole structure. On the other hand, the horizontal and vertical behaviour of the GL solution was almost decoupled.

More importantly, Figures 4 and 5 indicate that the DA solution (accounting for the axis offset from the ground level) captured the shear deformation profiles $\gamma$ (depending on the beam shear forces as shown in Equation (14)) of the experimental results, while providing limited bending deformations $\chi$ for both central and eccentric tunnel-structure configurations. Therefore, considering the offset $d_{a}$ increased the importance of shear, which was the primary response mechanism, with respect to bending deformations. Interestingly, the soil model $\mathrm{C} 2$ with additional decoupled springs provided greater $\gamma$ at the structure edges with respect to the $\mathrm{C} 1$ soil; this is in agreement with the centrifuge outcomes. In general, the strip footings under the end walls (modelled by $\mathrm{C} 2$ ) resulted in greater structure deformations ( $\gamma$ as well as $D R \mathrm{~s}$ ).

\section{The role of shear flexibility}

Extending beyond the centrifuge building geometries, the general effect of the shear flexibility on the deformation mechanisms of buildings is addressed in this section considering a range of equivalent anisotropic Timoshenko beams with varying $E / G$ ratios.

Three equivalent beams were investigated. Beams were specified to have a length of $B=20 \mathrm{~m}$, a Young's modulus, $E=1 \mathrm{GPa}$, a Poisson's ratio $v=0.3$, and rectangular cross-section of depth $d_{b}=H=9 \mathrm{~m}$ 
- $\mathrm{EXP}---\mathrm{GL}-\mathrm{C} 1-\mathrm{DA}-\mathrm{C} 1$
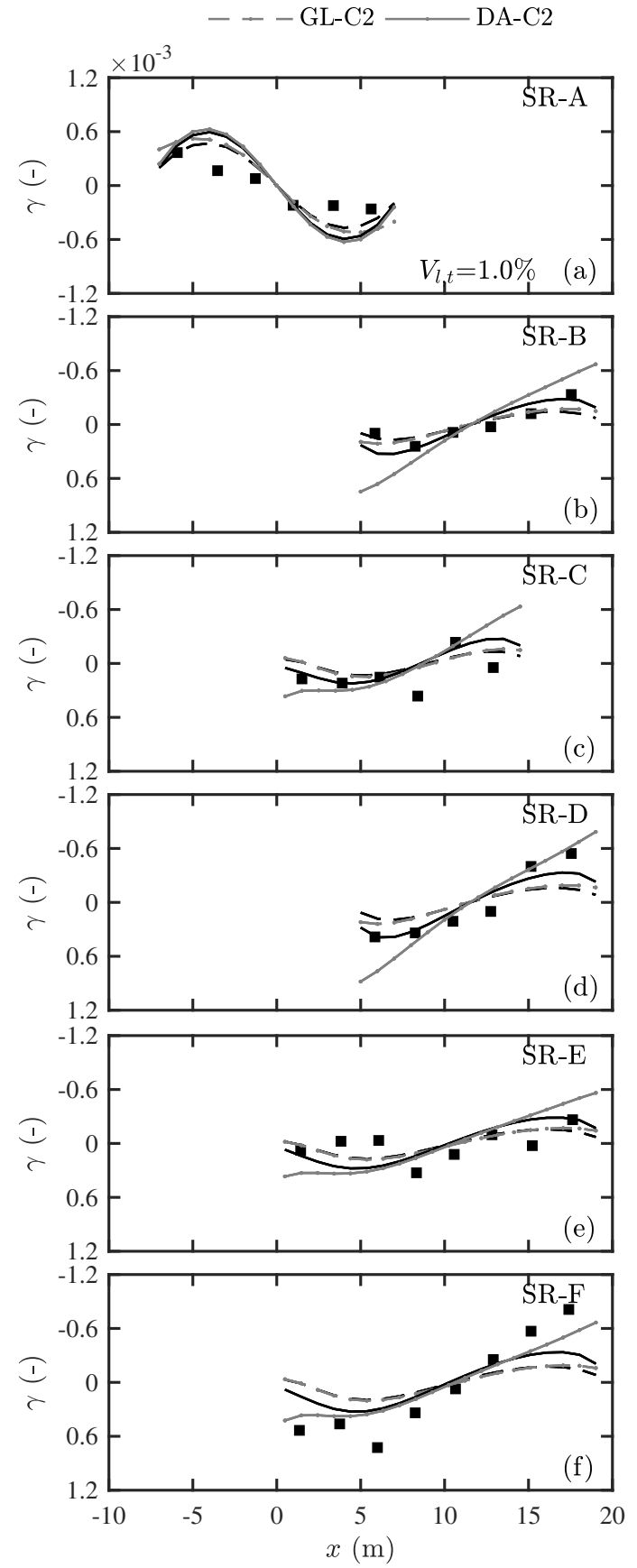

Figure 4: Shear strains: comparison with centrifuge data.

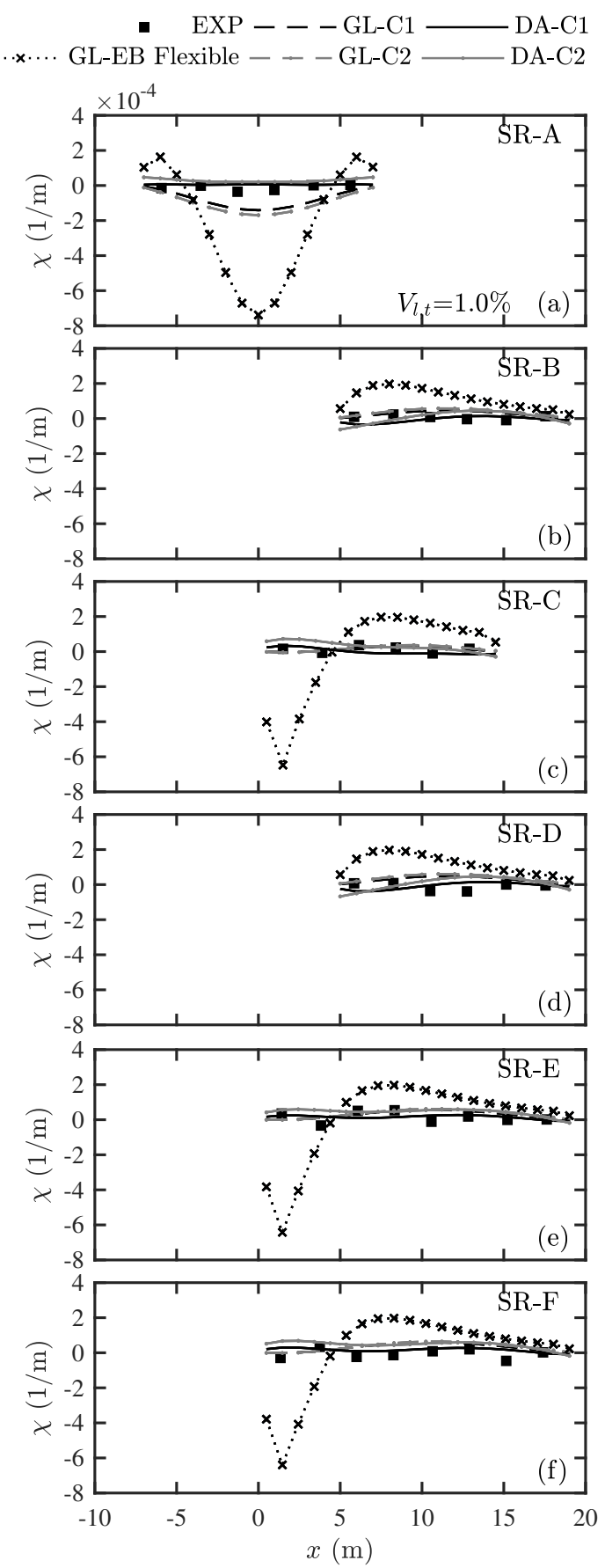

Figure 5: Curvature: comparison with centrifuge data. 
and width $b_{b}=L=0.5 \mathrm{~m}$. The structure axis was located at the beam mid-height. Three $E / G$ ratios were considered: $E / G=0,2.6$, and 12.5. $E / G=2.6$ corresponds to an isotropic material with $v=0.3$. $E / G=12.5$ represents low shear stiffness structures (e.g. because of the presence of openings as detailed by Burland et al. (1977)). The extreme case of the Euler-Bernoulli beam, a Timoshenko beam with $E / G=0$, is also of interest. In this section, the two Timoshenko beams are labelled as TIM\# where \# is the $E / G$ ratio, whereas the Euler-Bernoulli beam is labelled EB.

Regarding the geotechnical domain, two tunnelling scenarios were investigated: $6 \mathrm{~m}$ diameter $(D)$ tunnels with a depth to tunnel axis of $z_{t}=8 \mathrm{~m}$ and $20 \mathrm{~m}$. For each tunnelling case, two relative tunnel-structure eccentricity values $(e / B=0$ and 0.5$)$ were considered. Tunnelling-induced surface ground movements in greenfield conditions were defined on the basis of Equation (1) for $V_{l, t}=1 \%$. Finally, a soil Young's modulus $E_{s}=100 \mathrm{MPa}$ and a Poisson's ratio $v_{s}=0.5$ were assumed.

Tunnelling-induced vertical $\left(u_{z}\right)$ and horizontal $\left(u_{x}\right)$ displacements of the beam axes predicted by the elastic GL $\left(d_{a}=0\right)$ and DA $\left(d_{a}=H / 2\right)$ solutions are displayed in Figures 6 and 7 for $z_{t}=8$ and 20m, respectively. Note that structural displacements are reported at the height of the structure axis, i.e. $z=0$ and $z=-H / 2$ for GL and DA solutions, respectively. For comparison, greenfield settlements at the ground level $(z=0)$ are also reported (dotted lines).

Structure settlement curves are shown in Figures 6(a),(c) and 7(a),(c). Firstly, beams with their axis at the ground level (GL, solid lines) are discussed because their flexural and axial response is almost decoupled (i.e. horizontal ground movements marginally affect axis settlements and structure deflection does not significantly impact axial strains). Results confirm that EB beams are stiffer than TIM beams, which have additional shear flexibility. In addition, the shape of $u_{z}$ curves is worth noting. In Figure 6(a), EB beams tend to deform entirely in sagging (similarly to the greenfield curve), whereas the EB beam in Figure 7(a) also responds entirely in sagging but this is unlike the greenfield settlement curve. On the other hand, beams TIM2.6 and TIM12.5 have a deflection shape similar to the greenfield settlement trough when spanning across greenfield sagging and hogging zones (see Figures 7(a) and (c)), whereas their stiffness results in a hogging region at the structure edges and a narrow sagging region of the structure in Figure 6(a). In general, the increase in shear flexibility caused a variation in $i_{b l d}$. This mechanism impacted the structure deformations in terms of flexural modification factors, $M^{D R}$ and $M^{B}$, as discussed later in the text. On the other hand, horizontal displacement profiles $u_{x}$ for the EB and TIM solutions overlap in Figures 6(b) and $7(b)$.

Secondly, DA beams, which account for the offset between the ground level and the beam axis, are analysed. In addition to the interaction mechanisms characterising the GL solutions, the effect of the ground level-beam axis offset resulted in the stiffening of the DA structures with respect to GL beams, particularly for low shear flexibility structures (EB and TIM0.5). This stiffening effect caused the decrease of the maximum tunnelling-induced structure settlements of the DA beams and caused the EB beam in Figure 6(a) to have slightly negative relative deflections. These mechanisms are due to the fact that the offset $d_{a}$ results in the coupling of rotational and horizontal degrees of freedom (Equations (10a) and (10b), e.g. horizontal forces at the ground level induce distributed bending movements along the structure axis) and a soil stiffness matrix $\mathbf{K}_{\mathbf{s}}{ }^{*}$ at the beam axis with additional stiffness for the beam rotational terms (Cheung and Nag, 1968).

Bending deformations of the structures EB and TIM0.5 mobilised the stiffness of the matrix $\mathbf{K}_{\mathbf{s}}{ }^{*}$ at the rotational degrees of freedom and resulted in high compressive and tensile reaction forces of the soil for central and eccentric beams, respectively (as displayed in Figures 7(b) and 7(d) the DA structures undergo greater negative and positive horizontal strains than GL beams). On the other hand, the effects of the axis offset on tunnelling-induced displacements are minor for structures TIM2.6 and TIM12.5 because most of the deflection is induced with minor nodal rotations; for instance, $u_{z}$ curves of TIM12.5-DA are close to 

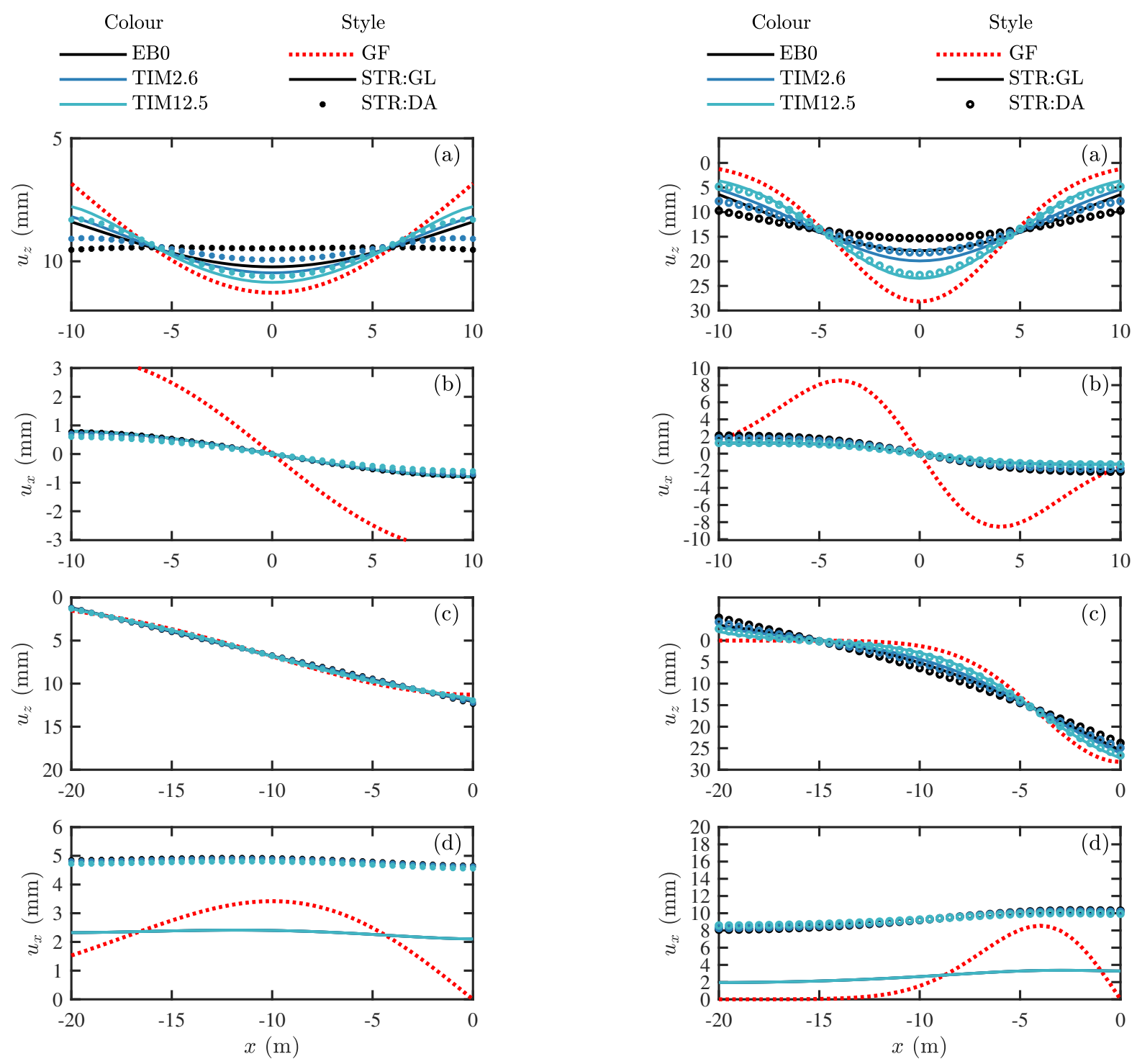

Figure 6: Greenfield and beam displacements for $z_{t}=20 \mathrm{~m}$ : (a) and (b) $e / B=0$; (c) and (d) $e / B=0.5$.

Figure 7: Greenfield and beam displacements for $z_{t}=8 \mathrm{~m}$ : (a) and (b) $e / B=0$; (c) and (d) $e / B=0.5$.

values associated with TIM12.5-GL at the ground level. Additionally, eccentric DA beams in Figures 6(d) and 7(d) display an additional horizontal shift towards the tunnel centreline with respect to GL beams resulting from the tilt of the structure (i.e. the cross-sectional rotations). Overall, in the DA solutions the influence of the offset $d_{a}$ was significant for beams mostly deforming in bending because of the low $E / G$ ratio.

Next, modification factors $M^{D R}$ and $M^{B}$ associated with GL solutions are presented in Figure 8. This figure displays that larger $E / G$ ratios (i.e. increased shear flexibility) cause larger $M^{D R}$ values both in sagging and hogging. Furthermore, $M^{B}$ tends to unity for high $E / G$ in the case of structures spanning across the greenfield sagging and hogging regions (for $z_{t}=8 \mathrm{~m}$ ), whereas for beams entirely located in the sagging zone $\left(z_{t}=20 \mathrm{~m}\right) M^{B, s a g} \leq 1$ and its value decreases with $E / G$. 

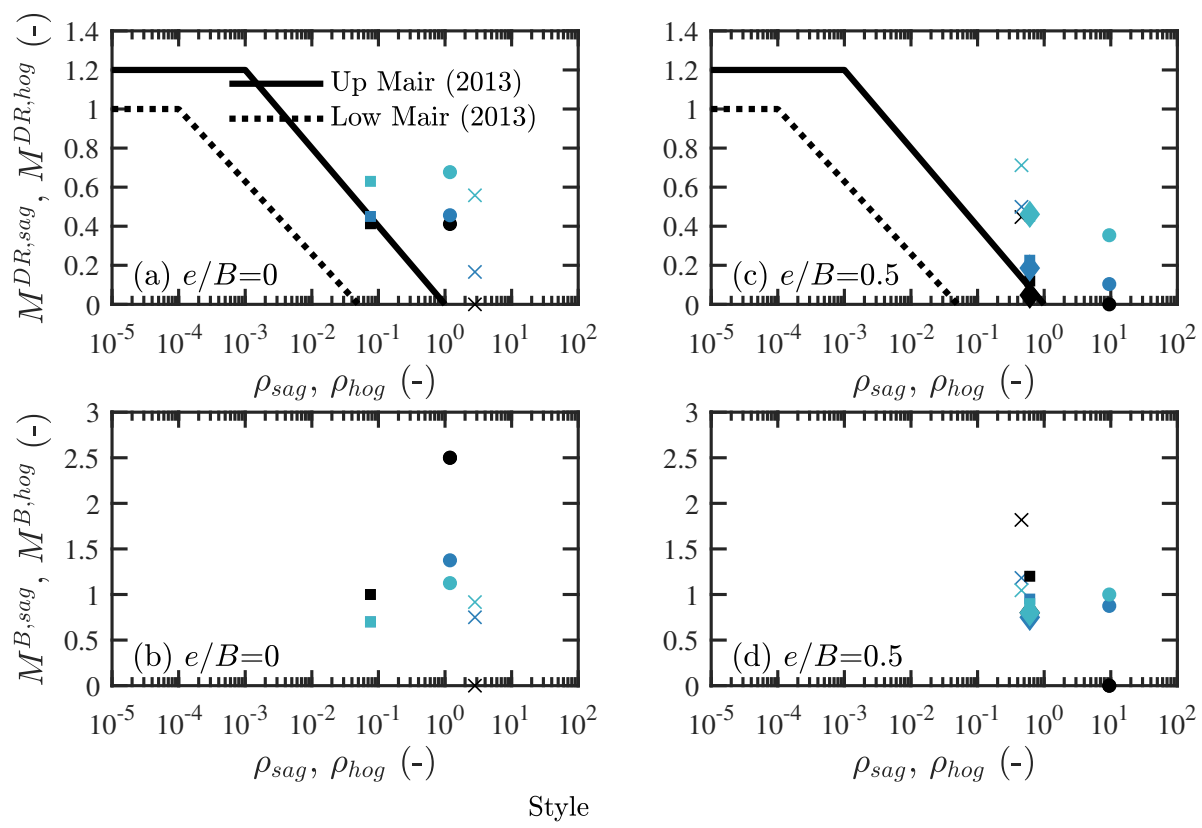

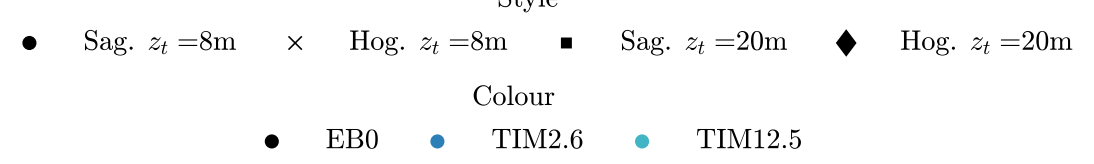

Figure 8: Modification factors for GL solutions of the simple beams.

To better distinguish between bending and shear deformations, internal forces ( $N=$ axial force, $S=$ shear force, $M=$ bending moment) and total bending $\left(\varepsilon_{b t}\right)$ and diagonal strains $\left(\varepsilon_{d t}\right)$ induced by the central tunnel with $z_{t}=8 \mathrm{~m}$ and $e / B=0$ at the simple beam axis are displayed in Figures 9 and 10 for GL and DA solutions, respectively.

For the GL solutions, Figure 9(a) illustrates that: [i] the entire structure is in sagging (bottom fibres are tensile) for $e / B=0$, despite the fact that the structures span across the greenfield sagging and hogging regions and the settlement troughs contain inflection points for Timoshenko beams with $E / G>0$ (see Figure 7), [ii] $S$ and $M$ decrease in magnitude with $E / G$ because of the increase in shear flexibility, [iii] the force profile shapes do not depend on the $E / G$ ratio. Meanwhile, Figure 9(b) shows that bending and shear deformations are dominant for low and high $E / G$ values, respectively, while the maximum diagonal strains occurred near the location of the greenfield inflection points.

The influence of the axis offset from the ground level is considered by comparing Figures 9 and 10. The DA beams are characterised by greater $N$ and $S$ forces while the magnitude of the bending moments $M$ is decreased with respect to the GL beams; this caused DA beams to be subjected to slightly higher total shear strains $\varepsilon_{d t}$ and significantly lower total bending deformations $\varepsilon_{b t}$ than GL beam models. In both Figures 9 and 10, EB beams are subjected to greater forces than TIM beams.

Next, internal forces and strains in Figures 9 and 10 are compared with the settlement troughs in Figure 7. As mentioned previously, the inflection points of the settlement trough correspond to zero curvature locations only for EB beams $(E / G=0)$, whereas inflection points can represent non-zero curvature for TIM beams. Therefore, for TIM beam models dividing the structure into sagging/hogging regions using the settlement trough (e.g. DBA) rather then internal forces or curvature measurements (e.g. DSBA) could be 

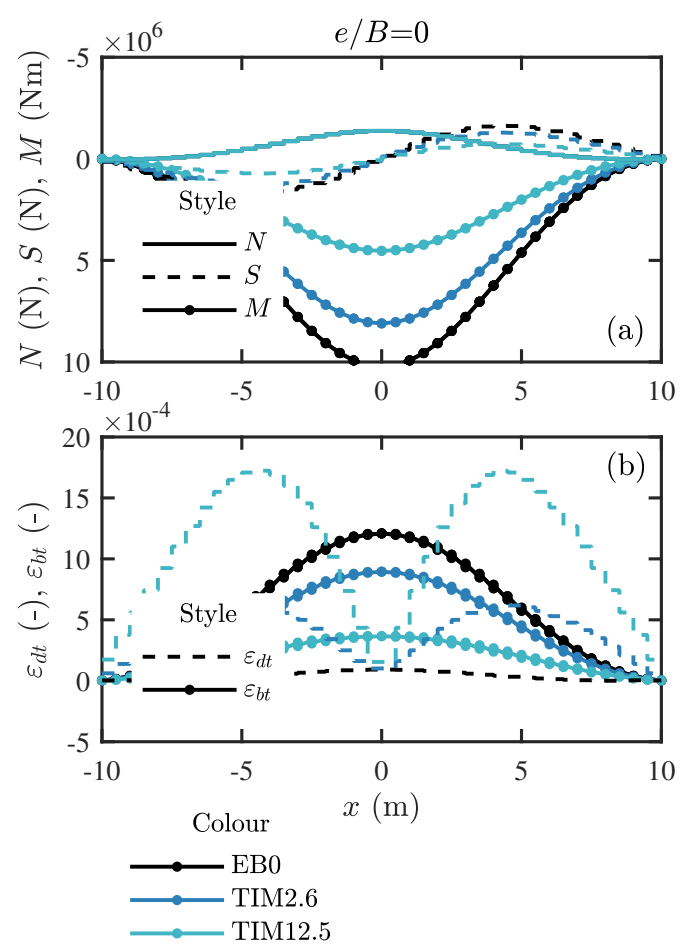

Figure 9: Beam response for the GL solution for $z_{t}=8 \mathrm{~m}$ : (a) inner forces; (b) total bending and diagonal strains.
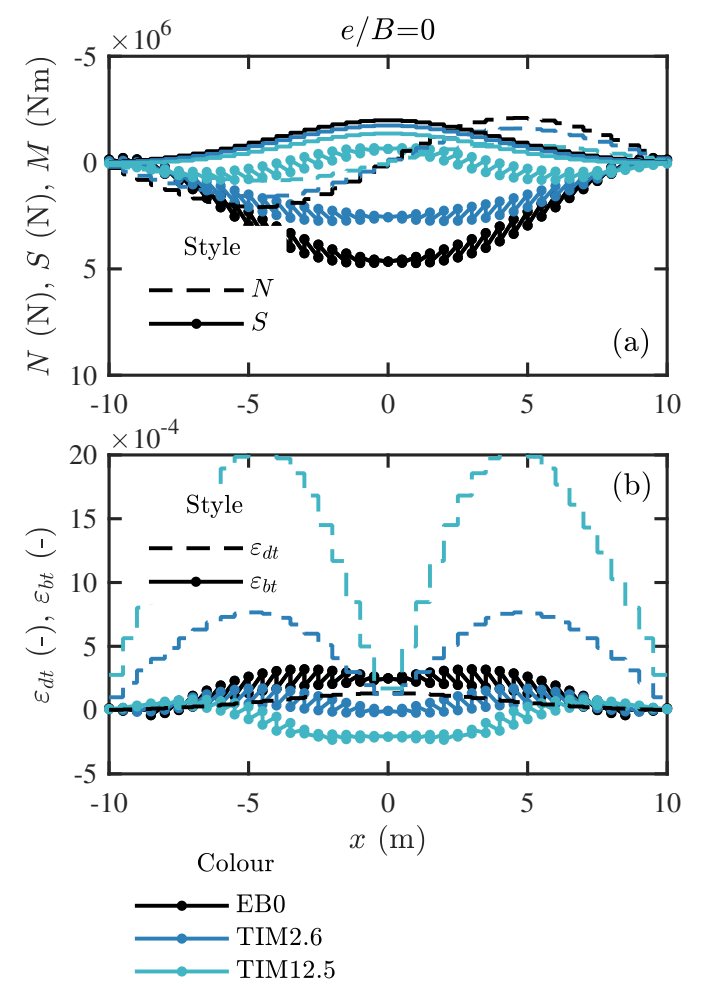

Figure 10: Beam response for the DA solution for $z_{t}=8 \mathrm{~m}$ : (a) inner forces; (b) total bending and diagonal strains.

misleading. Also note that, for TIM beams, bending strain due to bending moments occur at the inflection point of the settlement trough, which are not considered by Equation (8).

Finally, the total strains $\varepsilon_{b t}$ and $\varepsilon_{d t}$ predicted by the DSBA, DBA, and MBA for the considered anisotropic beams are compared in Figure 11 for both $e / B=0$ and 0.5 . Total bending strains $\varepsilon_{b t}$ predicted by the DBA and MBA do not agree well with the DSBA strains for structures with low shear flexibility $(E / G=0)$, whereas total diagonal strains $\varepsilon_{d t}$ predictions from the DBA and MBA differ from the DSBA strains for structures with high shear flexibility $(E / G=12.5)$. The scatter is limited for isotropic behaviour $(E / G=2.6)$. Therefore, although Equation (8) has been extensively used in tunnelling engineering with the DBA and the MBA, the direct strain approach DSBA may assess strains through Equation (14) with no need for further approximations.

\section{Relative structure-soil stiffness}

To derive a relative flexural structure-soil stiffness parameter accounting for both $E I$ and $G A_{s}$ values, it is assumed that a building subjected to a purely sagging/hogging settlement trough can be modelled as a Simply Supported (SS) Timoshenko beam loaded by either a central Concentrate Load (CL) or a uniform load (UL). For SS Timoshenko beams the structure deflection per unit load $(\delta / P)$ is inversely proportional to the normalised bending stiffness $E I /(1+a F)$ as follows 

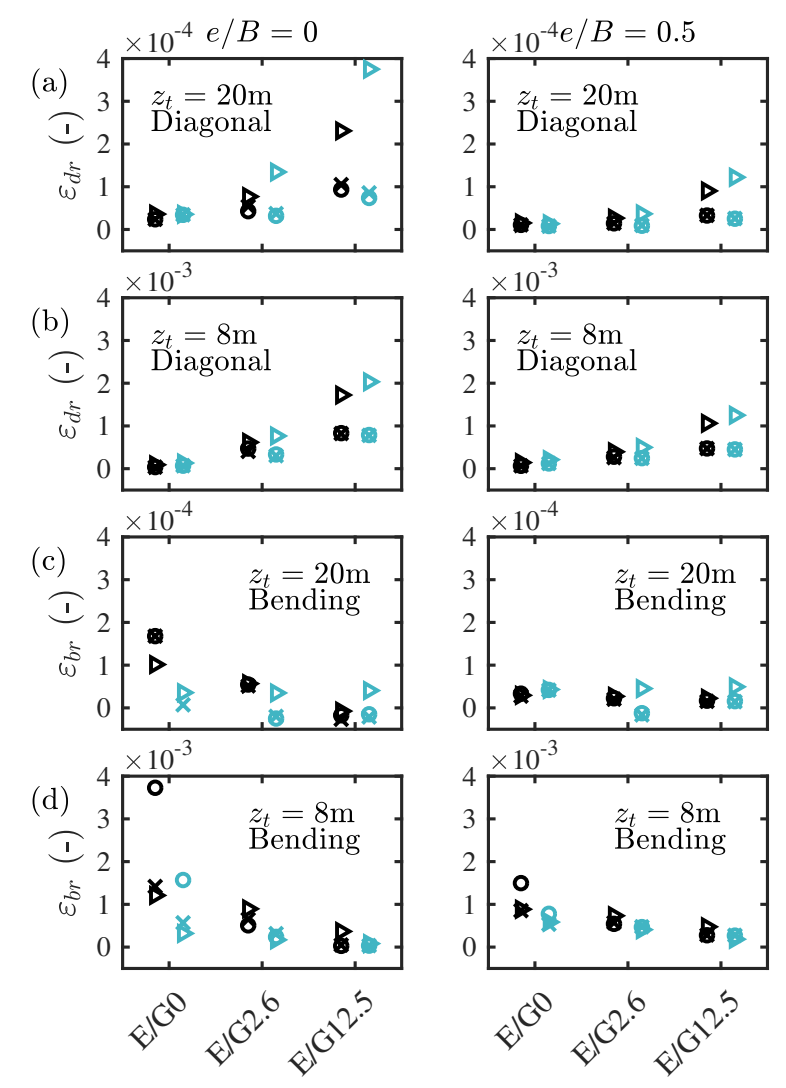

$$
\begin{array}{llll}
\times & \text { GL }- \text { DBA } & \times & \text { DA - DBA } \\
\text { ○ } & \text { GL - MBA } & \circ & \text { DA - MBA } \\
\triangleright & \text { GL - DSBA } & \triangleright & \text { DA - DSBA }
\end{array}
$$

Figure 11: Beam diagonal and shear strain for the DBA, MBA, and EBA approaches: (a) and (c) $z_{t}=20 \mathrm{~m}$, (b) and (d) $z_{t}=8 \mathrm{~m}$.

$$
\frac{\delta}{P}=b \frac{B^{3}}{\left(\frac{E I}{1+a F}\right)} ; \quad \frac{\delta^{\text {shear }}}{\delta^{\text {bending }}}=a F
$$

where $P$ is the total applied force, $B$ is the beam length with a sagging settlement profile, $F=(E I) /\left(B^{2} G A_{s}\right)$ is dimensionless, and the coefficients $a$ and $b$ depend only on the loading condition. Interestingly, the variation in $a$ and $b$ with the load scheme is limited: for CL and UL, $a=12$ and 9.6 whereas $b=8 / 384$ and 5/384, respectively. In general, $a$ and $b$ coefficients depend on the loading and boundary conditions (e.g. $a=3$ and $b=128 / 384$ are associated with a cantilever beam subjected to a concentrated load at the free-edge).

Importantly, $a F$ is the ratio between shear $\delta^{\text {shear }}$ and bending $\delta^{\text {bending }}$ deflections of the SS beam. For $F=1 / 25$ it follows that the shear deflection is about half of the bending settlement $(a F \approx 0.5)$ while for $F=1$ the deflection is dominated by shear deformations $(a F \approx 10)$. Therefore, the following threshold values are proposed: $F<1 / 25=$ bending-dominated behaviour, $F>1=$ shear-dominated behaviour, $1 / 25<F<1=$ mixed bending and shear response. For instance, for a bearing wall structure with no 
openings, $E / G=2.6$ and a rectangular cross-section, $F \approx(H / B)^{2} / 4$. For this structure, if the length-toheight ratio is $B / H=2.2$ then $F \approx 1 / 25$, whereas for a length-to-height ratio of $B / H=0.5, F \approx 1$.

For SS beams, an Euler-Bernoulli beam equivalent to the Timoshenko beam can be obtained by matching their central deflections, as shown in Equation (16). The equivalent bending stiffness of the EB beam $(E I)_{E B}$ relates to $E I$ of the TIM beam in terms of the reduction factor $\alpha^{\prime}=1 /(1+a F)$.

$$
(E I)_{E B}=\alpha^{\prime} E I=\frac{E I}{1+a F} ; \quad \alpha^{\prime}=\frac{1}{1+a F}
$$

To account for both the bending and shear cross-sectional stiffness, more general relative stiffness parameters $\eta$ can be written from Equations (5) and (16) as

$$
\eta_{\text {sag } / \text { hog }}=\frac{\rho_{\text {sag } / \text { hog }}}{1+a F_{\text {sag } / \text { hog }}}=\frac{\left(E I^{*}\right)}{E_{s} B_{\text {sag } / \text { hog }, g f}^{3}\left(1+a F_{\text {sag } / \text { hog }}\right)}
$$

where $a=12$, and $F_{\text {sag } / \text { hog }}=(E I) /\left(B_{\text {sag } / \text { hog,gf }}^{2} G A_{s}\right)$, so that $\eta_{\text {sag } / \text { hog }}=\rho_{\text {sag } / \text { hog }}$ for Euler-Bernoulli beams with $F_{\text {sag/hog }}=0$. To demonstrate the efficiency of Equation (17), two parametric studies were carried out adopting the GL elastic solution to investigate $M^{D R, s a g}$ and $M^{D R, h o g}$, which are referred to as 'flexural interaction parameters'. The greenfield settlement trough is given by a standard Gaussian curve, whereas the influence of tunnelling-induced horizontal movements and the offset between structure axis and ground level on these parameters is neglected. Analyses were carried out for greenfield $i=10 \mathrm{~m}$, soil stiffness $E_{s}=80 \mathrm{MPa}, v=0.3, v_{s}=0.5$, varying Young's modulus values for the simple beam and the soil, and varying $E I / G A_{s}$ ratios. For the considered Timoshenko beam, the foundation width is equal to the width of the building in the direction parallel to the tunnel, $b_{f}=b_{b}=L$.

Firstly, equivalent beams entirely located in the greenfield sagging and hogging zones were investigated $\left(D_{u} / i=+0.75, D_{l} / i=-0.75\right.$ for sagging structures; $D_{u} / i=2.5, D_{l} / i=1$ for hogging structures; $D_{u}$ and $D_{l}$ are maximum and minimum absolute offsets of the structure edges from the tunnel centreline, respectively, as shown in Figure 1). Modification factors $M^{D R}$ are plotted against $\rho$ and $\eta$ in Figure 12. Different plot styles are used to distinguish between $F$ values: $F=0$ results are for Euler-Bernoulli beams, whereas greater $F$ values are associated with greater shear deformations of Timoshenko beams. The effects of the building plan shape are displayed by comparing sub-plots(a)-(b), which are for $B / L=0.15$, to sub-plots(c)(d), which are for $B / L=15$. The lower the value of $B / L$, the closer the soil conditions are to plane-strain.

Figures 12(a) and (c) both show the expected decrease in the modification factor $M^{D R}$ with $\rho$. However, for high $F$ values the structure flexibility in shear is greater than for bending; thus, for high $F$ values $M^{D R}>0$ are entirely due to shear deformations while $M^{D R}$ decreases with the increment in $\rho$ because of the increase in the shear stiffness $G A_{s}$. Figures 12(a) and (c) also indicate that for a given $B / L$ ratio and $F$ value, both purely sagging and hogging structures are associated with almost identical $M^{D R}-\rho$ trends; additionally, the shift towards greater $\rho$ values of the Timoshenko beams with respect to the Euler-Bernoulli is proportional to $F$.

Meanwhile, results in Figures 12(b) and (d) illustrate the effectiveness of the relative structure-soil stiffness $\eta$ (Equation (17)) to represent the total structure stiffness with a close match of Euler-Bernoulli beam $(F=0)$ and Timoshenko beam $(F>0)$ results, which is in agreement with the SS Timoshenko beam representation of the problem. Interestingly, for the range $a=6-18$, which covers realistic tunnellinginduced internal force distributions, the variations of $\eta$ is small because of the semi-log scale. Furthermore, there is an agreement between the $\eta-M^{D R}$ data and the empirical envelopes $\rho-M^{D R}$ given by Mair (2013); interestingly, results for $B / L=15$ and 0.15 results are close to the upper and lower empirical bounds, respectively. This trend is also in agreement with the conclusions of Franzius et al. (2006) and Mirhabibi 
and Soroush (2013). Finally, also note that additional analyses were performed (not shown) by scaling problem dimensions with respect to varying tunnel depth and by changing the cross-sectional depth-towidth ratio. These simulations confirmed the suitability of the adopted parameters $B / L$ and $F$ to capture the modification factors.
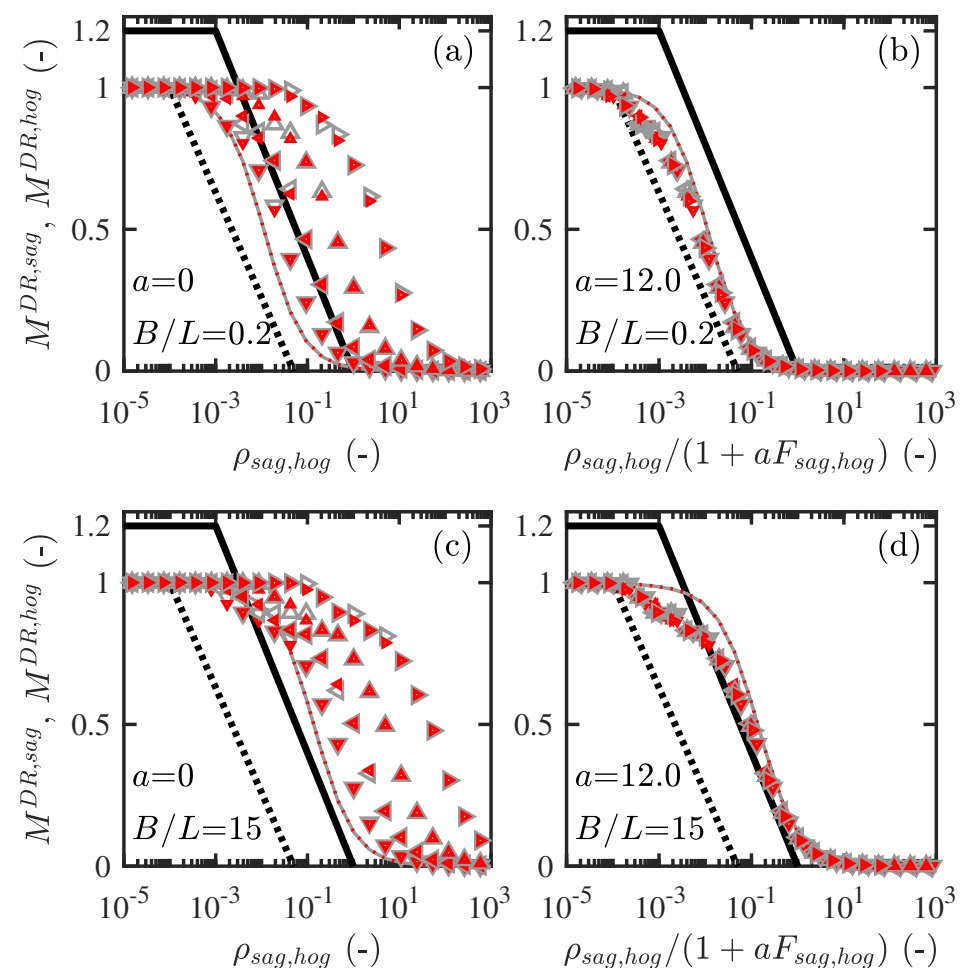

$\begin{array}{cl} & \text { Sag. } F=0 \\ \nabla & \text { Sag. } F=2.5 \\ \triangleleft & \text { Sag. } F=10 \\ \triangle & \text { Sag. } F=50 \\ \triangleright \quad & \text { Sag. } F=500\end{array}$

$$
\begin{array}{cl}
\ldots & \operatorname{Hog} \cdot F=2.5 \\
\triangleleft & \operatorname{Hog} \cdot F=10 \\
\triangleleft & \operatorname{Hog} \cdot F=50 \\
\triangleright & \operatorname{Hog} \cdot F=500
\end{array}
$$

Figure 12: Modification factors against relative stiffness calculated using Eq. (5) (a,c), Eq. (17) (b,d). Results are for pure sagging and pure hogging structures with axis at the ground level and no horizontal movements.

Additionally, the applicability of the hogging charts in Figure 12 to deep-excavation induced ground movements is considered. As illustrated by Mair (2013), the settlement profile due to deep-excavation in stiff clays can be characterised by the hogging portion of a standard Gaussian curve, while the structure response to deep-excavation ground settlements can be estimated with the relative stiffness parameter $\rho$. To consider pure hogging excavation-induced ground settlements and buildings at varying locations, the $M^{D R}-\eta$ curves for the structure locations given by $D_{u} / i=2.5, D_{l} / i=1$ (results in Figure 12) and $D_{u} / i=3.5, D_{l} / i=2$ (not shown) were compared; the building location had only a minor effect on results. Therefore, the $M^{D R}-\eta$ plots can also be used for deep-excavation-induced settlements.

Second, $M^{D R}$ values of equivalent beams spanning across the sagging and hogging regions of the settlement trough are investigate for $D_{l} / i=\left(-D_{u} / i,-1,0,+1\right)$ and $D_{u} / i=(2 ; 3)$, assuming $i=10 \mathrm{~m}$, $E_{s}=80 \mathrm{MPa}$, and $B / L=15$. Figures 13 (a) and (b) confirm the capability of $\eta$ to capture the shear flexibility contribution to the interaction problem. This is a consequence of the fact that the variation in the 
location of the inflection point of Timoshenko beams, estimated by $M^{B}$, only relate to the variation of the point of zero curvature and bending moment for bending dominated behaviour (as Euler-Bernulli beams).
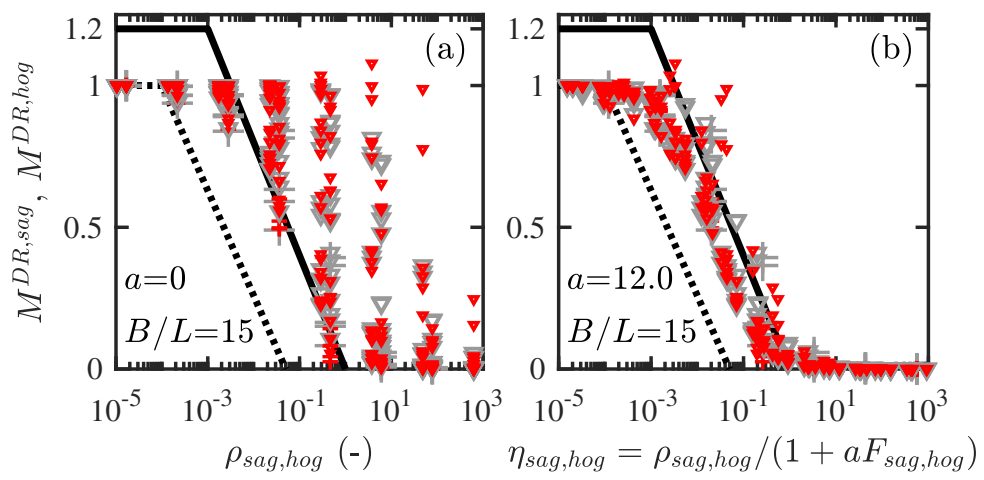

$+\quad$ Sag. $F<1 \quad+\quad$ Hog. $F<1$

$\nabla \quad$ Sag. $F>1 \quad \nabla \quad \operatorname{Hog} . F>1$

Figure 13: Modification factors against relative stiffness calculated using Eq. (5) (a) and Eq. (17) (b). Results are for structures spanning across sagging and hogging regions with axis at the ground level and no horizontal movements.

In conclusion, the foundation shape factor $(B / L)$ and the relative flexural stiffness parameter $\left(\rho_{\text {sag }}^{\prime} / \mathrm{hog}\right.$ for $F<1 / 25$ and $\eta_{\text {sag } / \text { hog }}$ for $F>1 / 25$ ) can be used to predict $M^{D R}$. To summarise the results of this work, a design chart is presented in Figure 14. The lower bound curve and the threshold value of 1.2 for the upper envelopes were selected after Mair (2013), while the upper bound is modified according to the results of this paper. This design chart still enables use of the existing framework proposed by Mair (2013), but improves it by adding one additional factor, the foundation shape factor $B / L$. However, this design chart still includes numerous assumptions so, when possible the direct strain method approach should be preferred to the use of the modification factor for risk assessments.

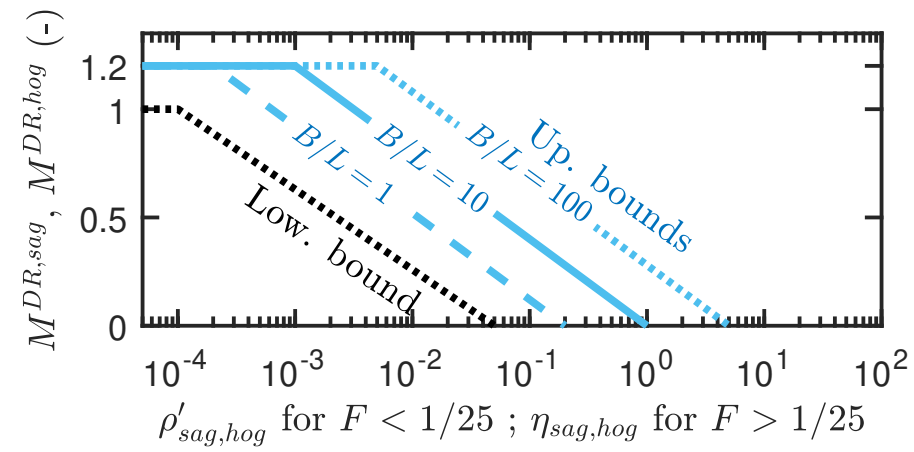

Figure 14: Design chart for the deflection ratio modification factor against relative stiffness, with the upper bound envelopes varying with the foundation shape factor. 


\section{Conclusions}

A Timoshenko beam model was incorporated into the FEM program ASRE to study tunnel-building interaction with coupled analyses. This tool enables efficient and rapid initial assessment of tunnellinginduced effects while accounting for soil-structure interaction. The following conclusions are drawn.

- The shear flexibility altered the response of the structure both in terms of the magnitude and distribution of displacements and deformations. Furthermore, as shown by comparison with centrifuge test data, Timoshenko beam models provided an accurate prediction of curvature and shear strains for wall bearing structures. Therefore, equivalent models of buildings with both bending stiffness $E I$ and shear stiffness $G A_{s}$ provide a better representation of the actual behaviour of the building than pure equivalent bending stiffness (with infinite shear stiffness).

- The offset between the axis of an equivalent beam and the ground level significantly impacts the strain prediction from the tunnel-structure interaction. This offset resulted in the coupling between flexural and axial deformations, which was more significant for lower shear flexibility (e.g. Euler-Bernoulli beams).

- Within the limiting tensile strain method, total bending and diagonal strains along the entire building can be inferred from either tunnelling-induced internal forces (obtained from the proposed coupled analysis method) or deformations parameters (measured in experiments and monitoring); this approach was named 'direct strain based approach' (DSBA). This overcomes the possible limitations of partitioning the structure in sagging and hogging zones, which resulted for several analysed cases in uncorservative tensile strain predictions. Alternatively, tunnelling-induced internal forces that can be used for forced-based risk assessments (e.g. Cai et al. (2017)).

- Based on the scheme of the simply supported Timoshenko beam, revised relative flexural structuresoil stiffness parameters that include the effect of both structural bending and shear stiffness are proposed.

- A design chart is proposed to estimate deflection ratio modification factors. It accounts for the effects of both the revised relative stiffness parameters and the foundation shape ratio.

\section{Acknowledgements}

This work was supported by the Engineering and PhysicalSciences Research Council (EPSRC) (EP/N509620/1).

The authors are grateful to Dr S. Ritter for sharing experimental data. 


\section{Appendix A}

\section{Timoshenko beams}

For equivalent Timoshenko beams with a rectangular cross-section and a generic target structure Equation (18) is obtained.

$$
E_{m}=E \frac{A_{0} \lambda}{b_{m} \sqrt{\frac{12 I_{0} \alpha}{A_{0} \lambda}}} \quad H_{m}=\sqrt{\frac{12 I_{0} \alpha}{A_{0} \lambda}} \quad G_{m}=G \frac{\kappa}{\kappa_{m}} \frac{A_{0} \beta}{b_{m} \sqrt{\frac{12 I_{0} \alpha}{A_{0} \lambda}}} \quad \text { or } \quad \frac{E_{m}}{G_{m}}=\frac{E}{G} \frac{\kappa}{\kappa_{m}} \frac{\lambda}{\beta}
$$

If the target structure façade is a homogeneous solid that has a rectangular cross-section (e.g. bearing wall resting directly on the soil surface), the beam model properties are given by Equation (19), for which $E_{m} / G_{m}$ would be close to the target material ratio $E / G$ (Pickhaver et al., 2010).

$$
E_{m}=E \frac{1}{\sqrt{\frac{\alpha}{\lambda}}} \lambda \quad H_{m}=H \sqrt{\frac{\alpha}{\lambda}} \quad G_{m}=G \frac{\beta}{\sqrt{\frac{\alpha}{\lambda}}} \quad \text { or } \quad \frac{E_{m}}{G_{m}}=\frac{E}{G} \frac{\lambda}{\beta}
$$

Previous research identified equivalent beam models by imposing $H_{m}=H$ (Son and Cording, 2005) that result in Equation (20). This equation imposes $\alpha=\lambda$, which can be erronous. Interestingly, Equation (20) quantifies the increase in $E_{m} / G_{m}$ induced by presence of openings when $H=H_{M}$ is assumed (Burland et al., 2004; Son and Cording, 2007).

$$
E_{m}=E \alpha \quad H_{m}=H \quad G_{m}=G \beta \quad \text { or } \quad \frac{E_{m}}{G_{m}}=\frac{E}{G} \frac{\alpha}{\beta}
$$

Finally, Franzius et al. (2006) and Maleki et al. (2011) defined the equivalent solid by matching the cross-sectional area and inertia while assuming $E=E_{m}$ and isotropic equivalent material $E_{m} / G_{m}=2(1+$ $v_{m}$ ), which results in Equation (21). This equation causes an erroneous estimate of the shear stiffness of the target structure when $G_{m} \alpha^{1 / 3} \neq G \beta$.

$$
\lambda=\alpha^{1 / 3} \quad H_{m}=H \alpha^{1 / 3} \quad G_{m}=\frac{E_{m}}{2\left(1+v_{m}\right)} \quad \text { or } \quad \frac{E_{m}}{G_{m}}=2\left(1+v_{m}\right)
$$

\section{Euler-Bernoulli beams}

Target structures are often simplified to isotropic equivalent beam/plate models with $H_{m}$ significantly lower than $H$ (Bilotta et al., 2017; Farrell et al., 2014). With a negligible shear flexibility, total stiffness is approximated by a pure bending behaviour $(E I)_{E B}$ (Goh and Mair, 2014; Haji et al., 2018b). Therefore, the bending reduction factor $\alpha^{\prime}$ to be applied to $E I_{E B}$ given by Melis and Rodriguez Ortiz (2001) accounts for both the increase in bending and shear flexibility given by openings, whereas Franza et al. (2019b) proposed to estimate $\lambda^{\prime}$ as $1-O$, where $O$ is the ratio of openings. The equivalent Euler-Bernoulli beam characteristics are

$$
E_{m}=E \frac{1}{\sqrt{\frac{\alpha^{\prime}}{\lambda^{\prime}}}} \lambda^{\prime} \quad H_{m}=H \sqrt{\frac{\alpha^{\prime}}{\lambda^{\prime}}} \quad \frac{E_{m}}{G_{m}} \approx 0
$$


Bilotta, E., Paolillo, A., Russo, G., Aversa, S., 2017. Displacements induced by tunnelling under a historical building. Tunnelling and Underground Space Technology 61 (Jan), 221-232.

Bloodworth, A. G., Houlsby, G. T., 2017. Analysis of pre-vault tunnelling interaction with buildings. Proceedings of the Institution of Civil Engineers - Geotechnical Engineering 170 (2), 99-116.

Boldini, D., Losacco, N., Bertolin, S., Amorosi, A., 2018. Finite Element modelling of tunnelling-induced displacements on framed structures. Tunnelling and Underground Space Technology 80 (Oct), 222-231.

Boscardin, M. D., Cording, E. J., 1989. Building response to excavation-induced settlement. Journal of Geotechnical Engineering 115 (1), 1-21.

Burland, J. B., Broms, B. B., De Mello, V. F. B., 1977. Behaviour of foundations and structures. In: Proceedings of the 9th International Conference on Soil Mechanics and Foundations Engineering. Vol. 2. Tokyo, pp. 495-546.

Burland, J. B., Mair, R. J., Standing, J. R., 2004. Ground performance and building response due to tunnelling. In: Jardine, R. J., Potts, D. M., Higgins, K. G. (Eds.), Advances in Geotechnical Engineering: The Skempton Conference - Proceedings of a Three Day Conference on Advances in Geotechnical Engineering, organised by the Institution of Civil Engineers. Vol. 1. Thomas Telford Services Ltd, London, United kingdom, pp. 291-344.

Cai, Y., Verdel, T., Deck, O., 2017. Using plane frame structural models to assess building damage at a large scale in a mining subsidence area. European Journal of Environmental and Civil Engineering, 1-24.

Cheung, Y. K., Nag, D. K., 1968. Plates and beams on elastic foundations-linear and non-linear behaviour. Géotechnique 18 (2), 250-260.

Cook, D., 1994. Studies of settlement and crack damage in old and new facades. In: Proc., 3rd Int. Masonry Conference., London, England. Vol. 6. pp. 203-211.

Deck, O., Singh, A., 2012. Analytical model for the prediction of building deflections induced by ground movements. International Journal for Numerical and Analytical Methods in Geomechanics 36 (1), 62-84.

Dimmock, P. S., Mair, R. J., 2008. Effect of building stiffness on tunnelling-induced ground movement. Tunnelling and Underground Space Technology 23 (4), 438-450.

El Kahi, E., Deck, O., Khouri, M., Mehdizadeh, R., Rahme, P., 2018. Étude de l'influence de la plasticité du sol sur la transmission des mouvements du sol affectant l'interaction sol-structure. Revue Française de Géotechnique (156), 4.

Elkayam, I., Klar, A., 2019. Nonlinear elasto-plastic formulation for tunneling effects on superstructures. Canadian Geotechnical Journal 56 (7), 956-969.

Farrell, R., Mair, R., Sciotti, A., Pigorini, A., 2014. Building response to tunnelling. Soils and Foundations 54 (3), $269-279$.

Franza, A., DeJong, M. J., 2019. Elastoplastic solutions to predict tunneling-induced load redistribution and deformation of surface structures. Journal of Geotechnical and Geoenvironmental Engineering 145 (4), 04019007.

Franza, A., Marshall, A. M., Zhou, B., 2019a. Greenfield tunnelling in sands: the effects of soil density and relative depth. Géotechnique 69 (4), 297-307.

Franza, A., Ritter, S., DeJong, M. J., 2019b. Continuum solutions of tunnel-building interaction and a modified framework for deformation prediction. Géotechnique (In press).

Franzius, J. N., Potts, D. M., Burland, J. B., 2006. The response of surface structures to tunnel construction. Proceedings of the ICE - Geotechnical Engineering 159 (1), 3-17.

Fu, J., Yu, Z., Wang, S., Yang, J., 2018. Numerical analysis of framed building response to tunnelling induced ground movements. Engineering Structures 158 (Mar), 43-66.

Giardina, G., DeJong, M. J., Mair, R. J., 2015. Interaction between surface structures and tunnelling in sand: Centrifuge and computational modelling. Tunnelling and Underground Space Technology 50 (Aug), 465-478.

Goh, K. H., Mair, R. J., 2014. Response of framed buildings to excavation-induced movements. Soils and Foundations 54 (3), 250-268.

Haji, T. K., Marshall, A. M., Franza, A., 2018a. Mixed empirical-numerical method for investigating tunnelling effects on structures. Tunnelling and Underground Space Technology 73 (Mar), 92-104.

Haji, T. K., Marshall, A. M., Tizani, W., 2018b. A cantilever approach to estimate bending stiffness of buildings affected by tunnelling. Tunnelling and Underground Space Technology 71 (Jan), 47-61.

Kassimali, A., Craddock, J. N., Matinrad, M., 1986. Bending and transverse shear stresses in fiber-composite beams by the transformed-section method. Composite Structures 5 (1), 33-49.

Losacco, N., Burghignoli, A., Callisto, L., 2014. Uncoupled evaluation of the structural damage induced by tunnelling. Géotechnique 64 (8), 646-656.

Mair, R., 2013. Tunnelling and deep excavations: ground movements and their effects. In: Anagnostopoulos, A., Pachakis, M., Tsatsanifos, C. (Eds.), Proceedings of the 15th European Conference on Soil Mechanics and Geotechnical Engineering - Geotechnics of Hard Soils - Weak Rocks (Part 4). IOS Press, Amsterdam, the Netherlands, pp. 39 - 70.

Mair, R. J., Taylor, R. N., Bracegirdle, A., 1993. Subsurface settlement profiles above tunnels in clay. Géotechnique 43 (2), $315-$ 320.

Mair, R. J., Taylor, R. N., Burland, J. B., 1996. Prediction of ground movements and assessment of risk of building damage due to bored tunnelling. In: Mair, R. J., Taylor, R. N. (Eds.), Proceedings of the International Symposium on Geotechnical Aspects of Underground Construction in Soft Ground. Balkema, Rotterdam, London, United Kingdom, pp. 713-718.

Maleki, M., Sereshteh, H., Mousivand, M., Bayat, M., 2011. An equivalent beam model for the analysis of tunnel-building interaction. Tunnelling and Underground Space Technology 26 (4), 524-533.

Marshall, A. M., Farrell, R., Klar, A., Mair, R., 2012. Tunnels in sands: the effect of size, depth and volume loss on greenfield 
displacements. Géotechnique 62 (5), 385-399.

Melis, M. J., Rodriguez Ortiz, J. M., 2001. Consideration of the stiffness of buildings in the estimation of subsidence damage by EPB tunnelling in the Madrid subway. In: Jardine, F. M. (Ed.), Proceedings of the international conference on response of buildings to excavation induced ground movements. Ciria Special Publication 201, London, UK, pp. 387-394.

Mirhabibi, A., Soroush, A., 2013. Effects of building three-dimensional modeling type on twin tunneling-induced ground settlement. Tunnelling and Underground Space Technology 38, 224-234.

O’Reilly, M. P., New, B. M., 1982. Settlements above tunnels in the United Kingdom - their magnitude and prediction. In: Jones, M. J. (Ed.), Tunnelling '82. London. IMM. pp. 173-181.

Peck, R. B., 1969. Deep excavations and tunnelling in soft ground. In: Proceedings of the 7th International Conference on Soil Mechanics and Foundation Engineering. Mexico City, Mexico, pp. 225-290.

Pickhaver, J., Burd, H., Houlsby, G., 2010. An equivalent beam method to model masonry buildings in 3D finite element analysis. Computers \& Structures 88 (19), 1049-1063.

Potts, D. M., Addenbrooke, T. I., 1997. A structure's influence on tunnelling-induced ground movements. Proceedings of the ICE - Geotechnical Engineering 125 (2), 109-125.

Ritter, S., 2017. Experiments in tunnel-soil-structure interaction. Ph.D. thesis, University of Cambridge.

Ritter, S., Giardina, G., DeJong, M. J., Mair, R. J., 2017. Influence of building characteristics on tunnelling-induced ground movements. Géotechnique 67 (10), 926-937.

Ritter, S., Giardina, G., DeJong, M. J., Mair, R. J., may 2018. Centrifuge modelling of building response to tunnel excavation. International Journal of Physical Modelling in Geotechnics 18 (3), 146-161.

Ritter, S., Giardina, G., Franza, A., DeJong, M. J., 2019. Building deformation caused by tunnelling: centrifuge modelling. Journal of Geotechnical and Geoenvironmental Engineering.

Son, M., 2015. Response analysis of nearby structures to tunneling-induced ground movements in sandy soils. Tunnelling and Underground Space Technology 48, 156-169.

Son, M., Cording, E. J., 2005. Estimation of Building Damage Due to Excavation-Induced Ground Movements. Journal of Geotechnical and Geoenvironmental Engineering 131 (2), 162-177.

Son, M., Cording, E. J., 2007. Evaluation of Building Stiffness for Building Response Analysis to Excavation-Induced Ground Movements. Journal of Geotechnical and Geoenvironmental Engineering 133 (8), 995-1002.

Vaziri, H., Simpson, B., Pappin, J. W., Simpson, L., 1982. Integrated forms of Mindlin's equations. Géotechnique 32 (3), $275-278$.

Yiu, W. N., Burd, H. J., Martin, C. M., 2017. Finite-element modelling for the assessment of tunnel-induced damage to a masonry building. Géotechnique 67 (9), 780-794. 

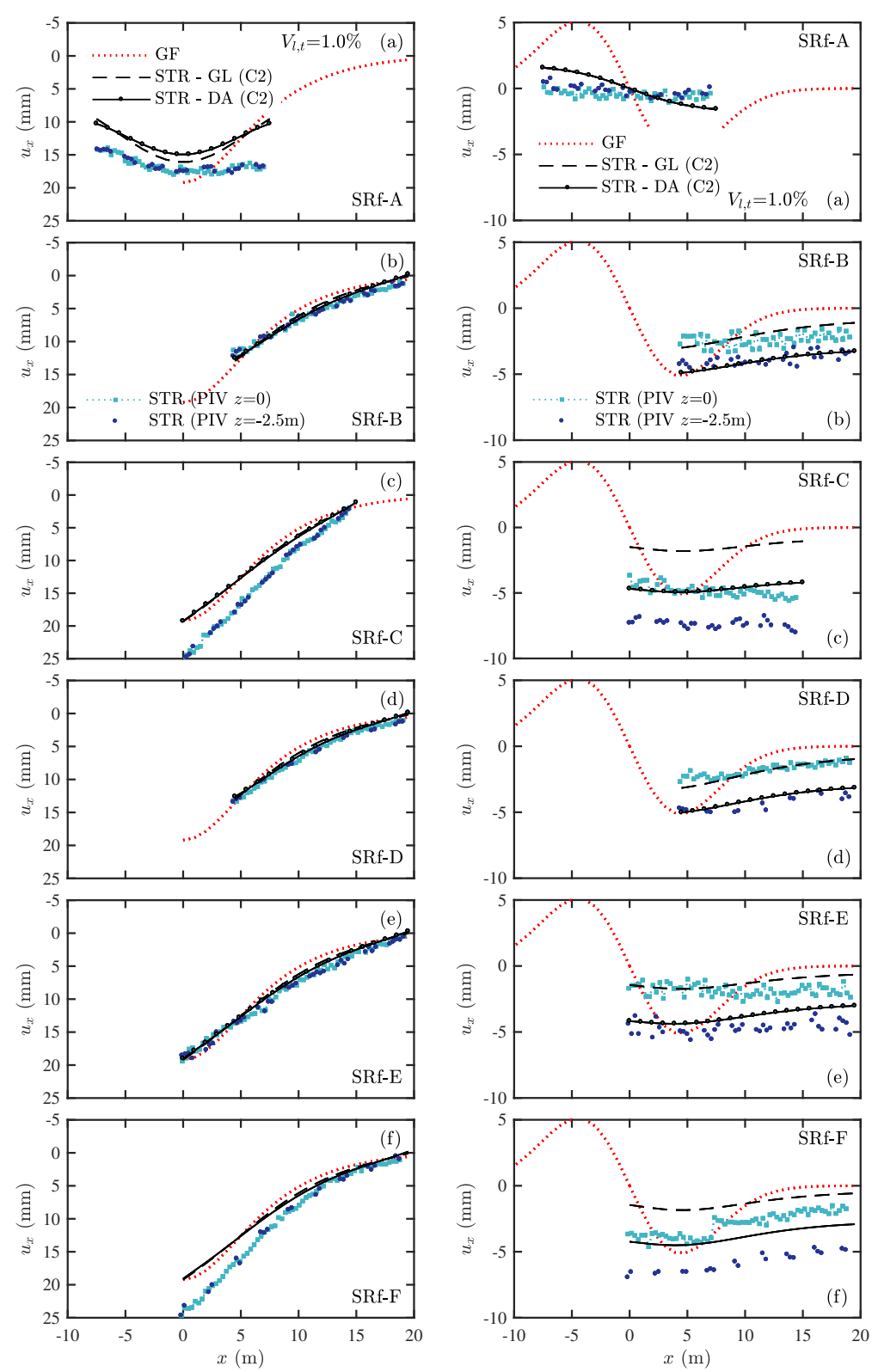

Figure S1: Comparison of DA and G1 solutions (for C2 soil model) with centrifuge testing results: (a-f) vertical and (g-m) horizontal displacements. 

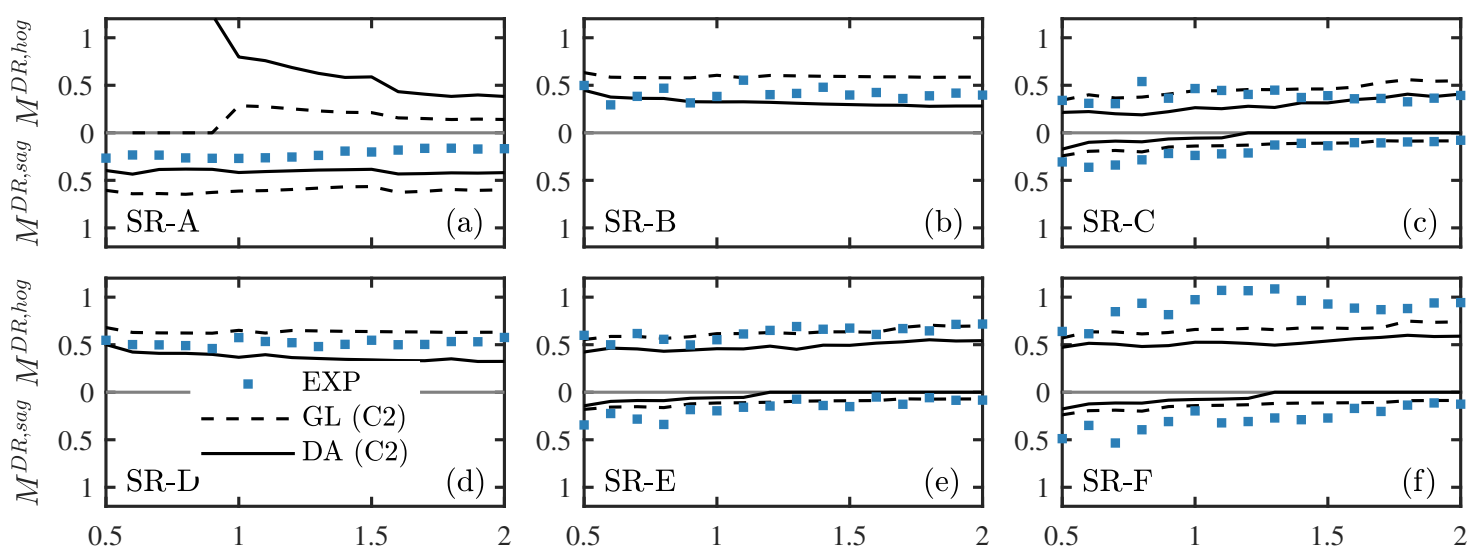

Figure S2: Comparison of DA and Gl solutions (for C2 soil model) with centrifuge testing results: deflection ratio modification factors 